\title{
Multibody systems with 3D revolute joints with clearances: an industrial case study with an experimental validation
}

\author{
Narendra Akhadkar ${ }^{\dagger}$. Vincent Acary ${ }^{\text {के }}$. Bernard Brogliato ${ }^{\text {क }}$
}

Received: date / Accepted: date

\begin{abstract}
This article is devoted to the analysis of the influence of the joint clearances in a mechanism of a circuit breaker, which is a forty-two degrees of freedom mechanism made of seven links, seven revolute joints, and four unilateral contacts with friction. Spatial (3D) revolute joints are modelled with both radial and axial clearances taking into account contact with flanges. Unilateral contact, Coulomb's friction and Newton impact laws are modeled within the framework of nonsmooth mechanics without resorting to some regularizations or compliance/damping at contact. The nonsmooth contact dynamics method based on an event-capturing time-stepping scheme with a second order cone complementarity solver is used to perform the numerical integration. Futhermore, the stabilization of the constraints at the position level is made thanks to the stabilized combined projected Moreau-Jean scheme. The nonsmooth modeling approach together with an event-capturing time-stepping scheme allows us to simulate, in an efficient and robust way, the contact and impacts phenomena that occur in joints with clearances. In particular, comparing with the event-detecting time-stepping schemes, the event-capturing scheme enables to perform the time-integration with large number of events (impacts, sliding/sticking transitions, changes in the direction of sliding) and possibly with finite time accumulations with a reasonable time-step length. Comparing with compliant contact models, we avoid stiff problems related with high stiffnesses at contact which generate some issues in contact stabilization and spurious oscillations during persistent contact periods. In the studied mechanisms of the circuit breakers, the numerical methods deals more than seventy contact points without any problems. Furthermore, the number of contact parameters is small : one coefficient of restitution and one coefficient of friction. Though they are sometimes difficult to measure accurately, the sensitivity of the simulation result with respect to contact parameters is low in the mechanism of the circuit breaker. It is demonstrated that this method, thanks to its robustness and efficiency allows to perform a sensitivity analysis using a Monte Carlo method. The numerical results are also validated by careful comparisons with experimental data which show a very good correlation.
\end{abstract}

Keywords joint clearance; unilateral constraints; Coulomb's friction; impacts; stabilized combined Moreau-Jean time-stepping scheme; sensitivity analysis; experimental validation; circuit breaker

\footnotetext{
†Schneider Electric, 28 Rue Henri Tarze, 38050 Grenoble, France. narendra.akhadkar@schneider-electric.com. ^INRIA Grenoble Rhône-Alpes and Laboratoire Jean Kuntzman, University Grenoble-Alpes, 655 avenue de l'Europe, 38334 Saint-Ismier, France. E-mail: narendra.akhadkar@schneider-electric.com, vincent.acary@inria.fr, bernard.brogliato@inria.fr
} 


\section{Introduction}

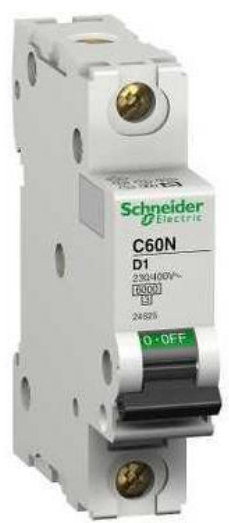

(a) External view

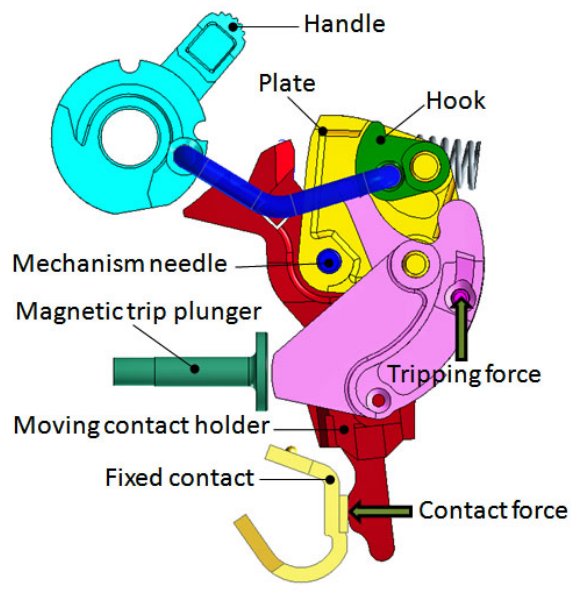

(b) View-1.

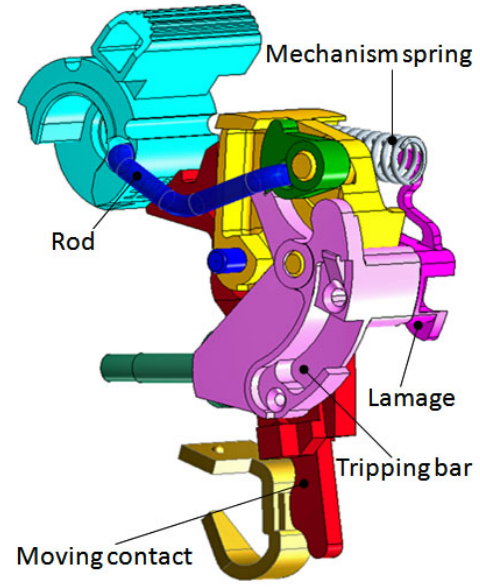

(c) View-2.

Fig. 1: Schneider Electric C-60 circuit breaker mechanism.

A miniature circuit breaker is a device that switches and/or protects the lowest common distributed voltage in an electrical system. A typical example of such a mechanism is the Schneider Electric's C-60 circuit breaker illustrated in Figure 1 It is designed to protect conductors and insulation from damage due to overload and short circuit. During normal service conditions, the mechanical and electrical components of the circuit breaker are at rest. It results in long periods of inactivity of the operating mechanism. However, the prerequisite is: when a fault occurs in the electrical network, the circuit breaker mechanism must respond quickly to react and break the circuit to clear the fault. Usually, the response time is in milliseconds. Any failure in clearing the fault may result in severe damage to the equipment or network and human safety is at stake. Therefore, the operating mechanism of the circuit breaker must have robust performance ensuring quick response to the situation.

Usually, the performance of these mechanisms is not as desired, due to the manufacturing tolerances on links, clearances in the joints and the assembly tolerances. The radial clearance in the revolute joints is a source of variability in the initial conditions of the system, and also of the degradation of the system's performance. The degradation of the system is always in the form of vibration, very high reaction forces at the joints, decrease of precision. Usually in the case of revolute joints, radial clearance is considered and, for simplification purpose, the axial clearance is not taken into account. The spatial revolute joint with clearance in both axial and radial direction adds five extra degrees of freedom into the system. Compared to planar mechanisms, spatial mechanisms generate more complicated functions with the same number of links. Most of the previous work is focused on the radial clearance in the planar revolute joints modeled with frictionless compliant contact models [25, 46, 24, 69, 71, 53, 71, 76, 8, 57, 16,70 with possibly flexible links, finite-element computations of the contact behavior and lubrication (see also [10, Table 1] and references therein for a review of recent literature for the slider-Crank mechanism). However more recently the combined influence of the axial and radial clearances in the revolute joint has been studied in [45, 52, 13,62,47]. These articles are the continuation of the pioneering research of Dhande and Chakraborty [22] who developed an analytical procedure for the analysis of spatial mechanisms with clearances and of Deck, Dubowsky et al. [23,37,21] who developed an analytical and experimental framework for the study of spherical and revolute joints with clearances. Brutti et al.[13] developed a numerical model of a 3D revolute joint with clearances with and without lubrication. The compliant contact model is calibrated with the help of finite element technique. Liu et al. [45] studied the dynamics and control of the spatial multibody system with clearance in the cylindrical joints using absolute coordinate based method. Yan et al. [62] conducted some experiments to find out the relative journal motions inside the bearing. Based on this experimental study, a model for spatial revolute joint with clearance in both the radial and axial directions is proposed. Bauchau et al. [9] used the finite element approach to model flexible multibody system and presented the approach to study the effect of clearance and lubrication in the planar and the spatial revolute joints. Another interesting works has been developed by 
Virlez et al. [73] to study the clearances in spatial mechanisms and especially, the link between the planet gears and the planet carrier in an automotive differential model Very recently, Marques et al. [47] proposed an enhanced compliant model for spatial revolute joint with clearances that takes into account the full geometric and kinematic properties of the clearance joint as well as the characteristic of the contacting materials.

Proper modelling of the joint clearances in multibody mechanical systems is required to predict the behaviour of real systems. Different contact/impact models and simulation tools are available to analyze the effect of joint clearance on the dynamical behavior of multibody systems. Some commercial software and open-source software packages are available in the market for such kind of mechanism simulation. However most of them work on compliant methods (both in the normal and the tangential directions), where sticking modes are not properly modelled due to regularization of Coulomb's law at zero tangential velocity. Another major drawback of regularization is that the numerical stabilization of contact forces and accelerations during the persistent contact phases, is not an easy task. Spurious oscillations may appear in the simulation of these contact modes. Haroun et al. [32], presented an experimental verification of the obtained simulation results of a slider crank mechanism with one clearance revolute joint. The simulation results were obtained using dynamic simulation software ADAMS (which uses Newton-Euler formulation). The contact force model is based on the model proposed in [42]. The aim of this study was to demonstrate the capability of the dynamic simulation software ADAMS in the presence of revolute joint with clearance. A similar study has been made in [79] to optimize the performance of the slider crank mechanism by using the Design of Experiment (DoE) approach. The proposed model allows to predict the influence of design parameter changes, in order to minimize contact forces, accelerations, and power requirements due to the existence of joint clearance. Brutti et al. [13], presented a computer-aided model of 3D revolute joint with clearance suitable for implementation in multibody dynamic solvers. The revolute joint has been modelled by introducing a nonlinear equivalent force system. The presence of an actual revolute joint with clearance can be simulated using a force element, which means that the kinematic constraint equations can be substituted by external force elements like springs and dampers. Analysis of revolute joints with clearance is based on the Hertzian contact force model and the simulation model is built using MSC.ADAMS. The influence of the crank speed on the dynamic behaviour of the slider crank mechanism is studied by the authors. Gummer et al. [30,31] presented the modelling of clearances in revolute joints with the use of circle-in-circle contact for the planar system using the simulation software RECURDYN. The dissipative (damping) component of the contact force in RECURDYN depends on the relative normal velocity of the contacting surfaces and the depth of penetration, however in the case of ADAMS it only depends on the depth of penetration. The friction model in RECURDYN is a regularized model, based on cubic law. Various geometries of a slider-crank mechanisms are used to simulate the clearance in the revolute joint.

Most of the mechanisms in the Schneider Electric company use frictional contacts to ensure the stability of the equilibrium of the mechanism in closed position. Unfortunately, the compliant models cannot correctly model the sticking condition. The dissipation when an impact occurs between two thermoplastic parts is high and again, compliant models are difficult to tune when nearly plastic impacts are involved. In the nonsmooth contact dynamics (NSCD) approach, the interaction of the colliding bodies is modelled with multiple frictional unilateral constraints [35, 48, 49,50,34]. The unilateral constraints are described by set-valued force laws in normal and tangential directions. The normal contact law is based on Signorini's condition at the velocity level including the Newton impact law, while the tangential contact law is based on Coulomb's friction law with a second order cone. Careful comparisons between numerical results obtained with the NSCD approach, and experimental data are reported in [40,63,65,66,67,68], while the use of the NSCD approach for systems with clearances is also advocated in [27,8]. They demonstrate that the numerical schemes and the model used in this article, possess very good forecast capabilities. Furthermore, we improve the standard NSCD method by a stabilization of the constraints at the position level [2] using a combined Moreau-Jean scheme that ensures the constraints both at the velocity and the position levels. This stabilization is mandatory when we deal with tight clearances.

Generally, the robust performance of the mechanism is achieved by allocating the dimensional tolerances on the parts, as small as possible. However the product cost increases significantly due to precise manufacturing process. Optimum balance between product cost, functionality, quality and time to market must be satisfied to ensure the competitiveness of the product. Usually the tolerance design method is used to manufacture the high-precision assemblies at lower costs [14,78]. In the tolerance analysis, the effect of the geometrical variations of the individual parts on the functional characteristics of the final assembled products are studied [72]. The method is extensively used in the manufacturing industry for improving the product quality and reliability (see [15, 22, 28, 29,36, 33, 51, 
58,77] for studies on tolerance analysis). There are two ways of tolerance analysis: either worst-case or statistical analysis. In the worst-case tolerance analysis, the worst possible combination of parts tolerances is considered (for example, shaft and hole assembly, in this case there are two possible worst cases, i.e. minimum diameter of the hole and the maximum diameter of shaft to produce the minimum clearance, and vice versa for the maximum clearance case). The important point to note is that it produces functional assemblies that meet functional requirements. The worst-case analysis ensures $100 \%$ interchangeability of the parts in an assembly [75]. However from a statistical point of view, the probability of such assemblies is null. The major limitation of the worst-case analysis is tight tolerances on the parts, and hence an increase of the production cost. In the statistical tolerance analysis, the desired product performance is achieved in a more economical way by allowing small percent of non-conformance, i.e. by allowing interchangeability less than $100 \%$. It does not focus on the extreme values of the part tolerances [60]. Usually in the statistical tolerance analysis, it is assumed that the part dimensions vary randomly according to a normal distribution. Also the normal distribution is centered at the midpoint of the total tolerance range with its $\pm 3 \sigma$ ( $\sigma$ denotes the standard deviation) spread covering the tolerance interval.

Our objective in this article is to address the robustness of the Schneider Electric C-60 miniature circuit breaker with respect to the production tolerances, which are expressed in the form of dimensional and geometrical variations on the parts. The aim is to study the influence of initial conditions and the out-of-plane motion, i.e. the polarization effec ${ }^{1}$ in the three dimensional case. Another objective is to develop a time efficien ${ }^{2}$ virtual test bench using the INRIA open-source simulation software SICONOS 3 in order to perform a sensitivity analysis via a Monte Carlo method. Our aim is to understand the influence of clearance in the revolute joints on the overall behavior of the C-60 miniature circuit breaker where human safety must be guaranteed. To validate the simulation model, experiments are carried-out on the prototype samples and the results are compared with the simulations.

The article is organized as follows. In Section 2 we introduce the dynamical equations which are used subsequently for the simulations and we describe the numerical time-integration scheme. Section 3 is dedicated to the detailed description of the C-60 mechanism. Section 4 contains the sensitivity analysis based on a Monte Carlo method. In Section 5, the numerical results are compared with experimental data. Conclusions are in Section 6 , and some details may be found in Appendix A

\section{Modeling and simulation of dynamics with unilateral contact, Coulomb friction and impact}

\subsection{Normal and tangential contact laws}

We assume that a local frame is defined by $\left(\mathrm{N}, \mathrm{T}_{1}, \mathrm{~T}_{2}\right)$ at a potential contact point between two bodies (see Figure 22. The gap $g_{\mathrm{N}}$ is defined as the signed distance between the two potential contacting points $C_{A}$ and $C_{B}$. The contact force, denoted by $r$, is decomposed in the local frame as $r=r_{\mathrm{N}} \mathrm{N}+r_{\mathrm{T}_{1}} \mathrm{~T}_{1}+r_{\mathrm{T}_{2}} \mathrm{~T}_{2}, r_{\mathrm{N}} \in \mathbb{R}, r_{\mathrm{T}}=\left[r_{\mathrm{T}_{1}}, r_{\mathrm{T}_{2}}\right]^{\top} \in$ $\mathbb{R}^{2}$. The contact local velocity is decomposed as well as $u=u_{\mathrm{N}} \mathrm{N}+u_{\mathrm{T}_{1}} \mathrm{~T}_{1}+u_{\mathrm{T}_{2}} \mathrm{~T}_{2}, u_{\mathrm{N}} \in \mathbb{R}, u_{\mathrm{T}}=\left[u_{\mathrm{T}_{1}}, u_{\mathrm{T}_{2}}\right]^{\top} \in \mathbb{R}^{2}$. Due to the impenetrability assumption one has $g_{\mathrm{N}} \geqslant 0$. We also neglect adhesive effects so that $r_{\mathrm{N}} \geqslant 0$. If $r_{\mathrm{N}}>0$ then we impose $g_{\mathrm{N}}=0$, and when $g_{\mathrm{N}}>0$, the normal contact force must vanish, i.e. $r_{\mathrm{N}}=0$ (no magnetic or distance forces) [1,4,11]. These conditions yield a complementarity condition, also termed as Signorini condition, denoted compactly as:

$$
0 \leqslant g_{\mathrm{N}} \perp r_{\mathrm{N}} \geqslant 0 .
$$

The normal contact law at the velocity level is expressed as :

$$
0 \leqslant u_{\mathrm{N}} \perp r_{\mathrm{N}} \geqslant 0, \quad \text { if } g_{\mathrm{N}}=0 \text {. }
$$

\footnotetext{
1 The polarization effect is created by two aspects: the presence of radial clearance in the revolute joint and the forces acting on the parts.
}

Definition 1 Polarization is the contact position between the two parts under the influence of an external force in an equilibrium stage.

\footnotetext{
2 To provide an example: a commercial software such as LS-DYNA (which is based on a compliant method) takes around six to eight hours to simulate the $\mathrm{C}-60$ breaker mechanism with clearance in the revolute joints on the standard desktop computer.

3 http://siconos.gforge.inria.fr/
} 


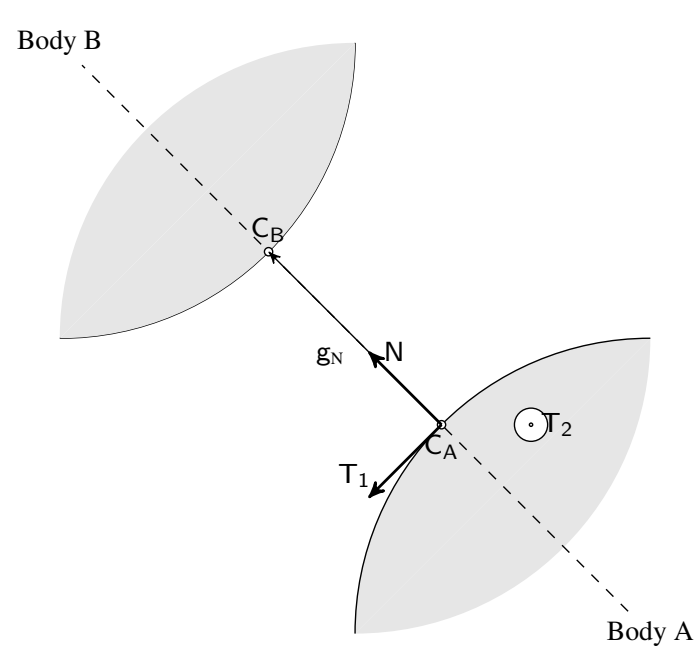

Fig. 2: Contact local frame.

The tangential contact law is the Coulomb friction which constrains the contact force $r$ in the second order friction cone (see Figure 3 (a)) :

$$
r \in K=\left\{r \in \mathbb{R}^{3},\left\|r_{\mathrm{T}}\right\| \leqslant \mu r_{\mathrm{N}}\right\}
$$

The scalar $\mu \geqslant 0$ is the coefficient of friction. In case of sliding (see Figure 3 $(\mathrm{b})$ ), the tangential force $r_{\mathrm{T}}$ acts in a direction opposite to the relative tangential velocity $u_{\mathrm{T}}$. If the relative tangential velocity $u_{\mathrm{T}}$ is zero then the bodies stick to each other (rolling without slipping). This yields a disjunctive formulation of the Coulomb friction as [4, 6, 5]:

$$
\left\{\begin{array}{llr}
r=0 & \text { if } g_{\mathrm{N}}>0 & \text { (no contact) } \\
r=0, u_{\mathrm{N}} \geqslant 0 & \text { if } g_{\mathrm{N}}=0 \quad \text { (take-off) } \\
r \in K, u=0 & \text { if } g_{\mathrm{N}}=0 \quad \text { (sticking) } \\
r \in \partial K, u_{\mathrm{N}}=0, \exists \beta>0, u_{\mathrm{T}}=-\beta r_{\mathrm{T}} & \text { if } g_{\mathrm{N}}=0 & \text { (sliding) }
\end{array}\right.
$$

Introducing the modified relative velocity $\widehat{u}:=u+\mu\left\|u_{\mathrm{T}}\right\| \mathrm{N}$ due to [20], the Coulomb friction can be equivalently expressed as a second-order cone complementarity condition [20,6] if $g_{\mathrm{N}}=0$ :

$$
K^{*} \ni \widehat{u} \perp r \in K \text {. }
$$

The cone $K^{*}$ is the dual cone of $K$, i.e., $K^{*}=\left\{z \in \mathbb{R}^{3} \mid z^{T} x \geqslant 0\right.$ for all $\left.x \in K\right\}$. Note that the basic Coulomb law can be easily enhanced with static and dynamic friction coefficients, varying friction coefficient (with Stribeck effects), or micro-displacements during sticking modes, while staying in a set-valued context that is suitable for a proper time-discretization including sticking modes [4, §3.9].

When a contact is closing with a negative relative normal velocity, we apply the Newton impact law:

$$
u_{\mathrm{N}}^{+}=-e_{r} u_{\mathrm{N}}^{-}, \text {if } g_{\mathrm{N}}=0 \text { and } u_{\mathrm{N}}^{-} \leqslant 0,
$$

where $u_{\mathrm{N}}^{+}$is the normal relative velocity after the collision, $u_{\mathrm{N}}^{-}$is the normal relative velocity before the collision, and $e_{r} \in[0,1]$ is the restitution coefficien 4 . We will see that the Newton impact law can be incorporated to the Signorini condition at the velocity level (2) if we formulate the law with the help of impulses.

\footnotetext{
4 When friction is present during impacts, there is in general no reason that $e_{r}$ should be upper bounded by 1, see [11 Chapter 4].
} 


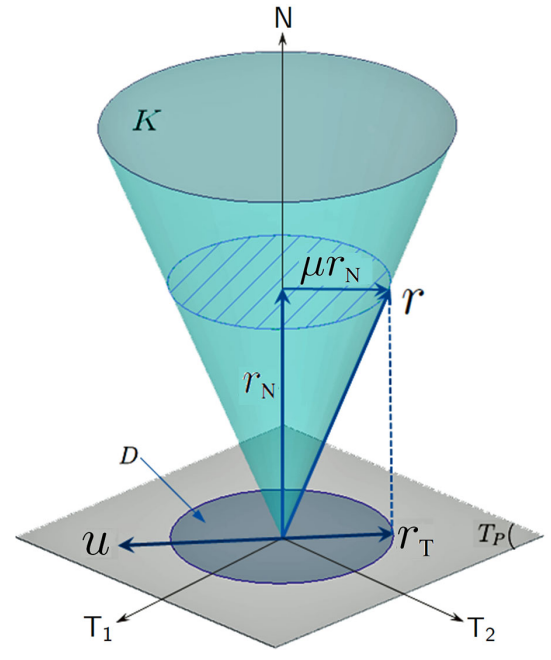

(a) 3D Coulomb's friction cone, the sliding case.

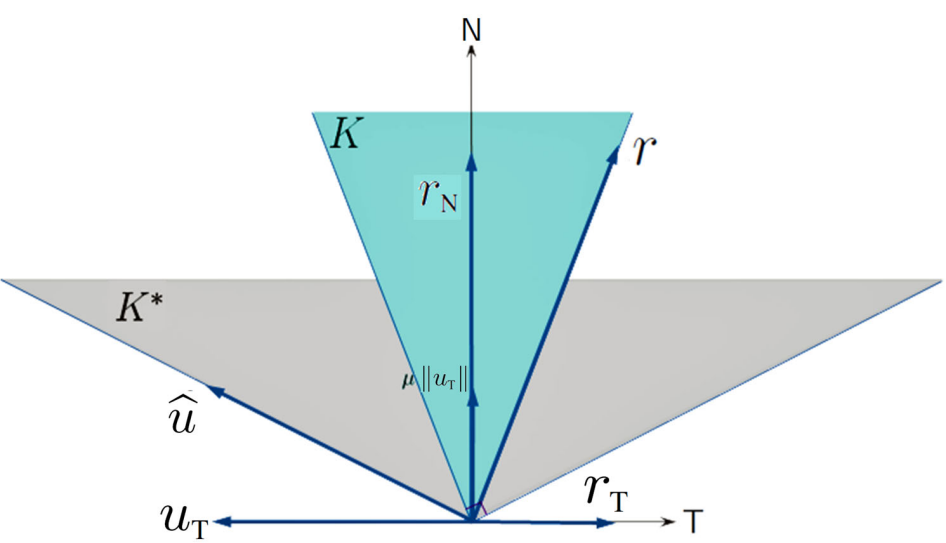

(b) Sliding case with modified velocity $\widehat{u}, r \in \partial K$.

Fig. 3: Coulomb's Friction law.

\subsection{Newton-Euler formulation of the equation of motion}

In this paper, we choose a maximal coordinates description of the kinematic of the mechanical system. This choice is motivated by the fact that it facilitates a change of modelling from an ideal joint to a joint with clearances. Indeed, it amounts to replacing a set of bilateral constraints by a set of unilateral constraints with friction, keeping the same set of kinematic variables. With a minimal coordinates system, and a possibly recursive formulation, a complete reformulation of the dynamical equation is necessary when a ideal joint is changed to a joint with clearances.

In the maximal coordinates framework, the most natural choice for the kinematic variables and for the formulation of the equations of motion is the Newton/Euler formalism, where the equation of motion describes the translational and rotational dynamics of each body using a specific choice of parameters. For the translational motion, the position of the center of mass $x_{\mathrm{g}} \in \mathbb{R}^{3}$ and its velocity $v_{\mathrm{g}}=\dot{x}_{\mathrm{g}} \in \mathbb{R}^{3}$ are usually chosen. For the rotational motion, a common choice is to choose the angular velocity $\Omega \in \mathbb{R}^{3}$ of the body expressed in the bodyfixed frame. The orientation of the body is usually defined by the rotation matrix $R$ of the body-fixed frame with respect to a given inertial frame. The angular velocity can be then expressed as :

$$
\widetilde{\Omega}=R^{\top} \dot{R}, \text { or equivalently, } \dot{R}=R \widetilde{\Omega},
$$

where the matrix $\widetilde{\Omega} \in \mathbb{R}^{3 \times 3}$ is given by $\widetilde{\Omega} x=\Omega \times x$ for all $x \in \mathbb{R}^{3}$. Using these coordinates, the equations of motion are given by

$$
\left\{\begin{aligned}
m \dot{v}_{\mathrm{g}} & =f\left(t, x_{\mathrm{g}}, v_{\mathrm{g}}, R, \Omega\right) \\
I \dot{\Omega}+\Omega \times I \Omega & =M\left(t, x_{\mathrm{g}}, v_{\mathrm{g}}, R, \Omega\right) \\
\dot{x}_{\mathrm{g}} & =v_{\mathrm{g}} \\
\dot{R} & =R \widetilde{\Omega}
\end{aligned}\right.
$$

where $m>0$ is the mass, $I \in \mathbb{R}^{3 \times 3}$ is the matrix of moments of inertia around the center of mass and the axis of the body-fixed frame. The vectors $f(\cdot) \in \mathbb{R}^{3}$ and $M(\cdot) \in \mathbb{R}^{3}$ are the total forces and torques applied to the body.

In the numerical practice, the choice of the rotation matrix is not convenient since it introduces redundant parameters. Since $R$ must belong to $S O^{+}(3)$, we have also to satisfy $\operatorname{det}(R)=1$ and $R^{-1}=R^{\top}$. In this work we choose to parametrize the rotation with a unit quaternion $p \in \mathbb{R}^{4}$ such that $R=\Phi(q)$ and $\dot{p}=\Psi(p) \Omega$. This parameterization has no singularity and has only one redundant variable that is determined by imposing $\|p\|=1$. Formulae for $\Phi$ and $\Psi$ can be found in any textbooks. We denote by $q$ the vector of coordinates of the position and 
the orientation of the body, and by $v$ the body twist:

$$
q:=\left[\begin{array}{c}
x_{\mathrm{g}} \\
p
\end{array}\right], \quad v:=\left[\begin{array}{c}
v_{\mathrm{g}} \\
\Omega
\end{array}\right] .
$$

The relation between $v$ and the time derivative of $q$ is

$$
\dot{q}=\left[\begin{array}{c}
\dot{x}_{\mathrm{g}} \\
\Psi(p) \dot{p}
\end{array}\right]=\left[\begin{array}{cc}
I & 0 \\
0 & \Psi(p)
\end{array}\right] v:=T(q) v
$$

with $T(q) \in \mathbb{R}^{7 \times 6}$. Note that the twist $v$ is not directly the time derivative of the coordinate vector as a major difference with Lagrangian systems. The Newton-Euler equation in compact form may be written as:

$$
\left\{\begin{array}{l}
\dot{q}=T(q) v \\
M \dot{v}=F(t, q, v)
\end{array}\right.
$$

where $M \in \mathbb{R} 6 \times 6$ is the total inertia matrix

$$
M:=\left(\begin{array}{cc}
m I_{3 \times 3} & 0 \\
0 & I
\end{array}\right),
$$

and $F(t, q, v) \in \mathbb{R}^{6}$ collects all the forces and torques applied to the body

$$
F(t, q, v):=\left(\begin{array}{c}
f\left(t, x_{\mathrm{g}}, v_{\mathrm{g}}, R, \Omega\right) \\
I \Omega \times \Omega+M\left(t, x_{\mathrm{g}}, v_{\mathrm{g}}, R, \Omega\right)
\end{array}\right) .
$$

When a collection of bodies is considered, we will use the same notation as in (11) extending the definition of the variables $q, v$ and the operators $M, F$ in a straightforward way.

2.3 Mechanical systems with bilateral and unilateral constraints

Let us consider that the system (11) is subjected to $m$ constraints, with $m_{e}$ holonomic bilateral constraints

$$
h^{\alpha}(q)=0, \alpha \in \mathcal{E} \subset \mathbb{N},|\mathcal{E}|=m_{e},
$$

and $m_{i}$ unilateral constraints

$$
g_{\mathrm{N}}^{\alpha}(q) \geqslant 0, \alpha \in \mathcal{I} \subset \mathbb{N},|\mathcal{I}|=m_{i} .
$$

Let us denote as $J_{h}^{\alpha}(q)=\nabla_{q}^{\top} h^{\alpha}(q)$ the Jacobian matrix of the bilateral constraint $h^{\alpha}(q)$ with respect to $q$ and as $J_{g_{\mathrm{N}}}^{\alpha}(q)$ respectively for $g_{\mathrm{N}}^{\alpha}(q)$. The bilateral constraints at the velocity level can be obtained as:

$$
0=\dot{h}^{\alpha}(q)=J_{h}^{\alpha}(q) \dot{q}=J_{h}^{\alpha}(q) T(q) v:=H^{\alpha}(q) v, \quad \alpha \in \mathcal{E} .
$$

By duality and introducing a Lagrange multiplier $\lambda^{\alpha}, \alpha \in \mathcal{E}$, the constraint generates a force applied to the body equal to $H^{\alpha, \top}(q) \lambda^{\alpha}$. For the unilateral constraints, a Lagrange multiplier $\lambda_{\mathrm{N}}^{\alpha}, \alpha \in \mathcal{I}$ is also associated and the constraints at the velocity level can also be derived as

$$
0 \leqslant \dot{g}_{\mathrm{N}}^{\alpha}(q)=J_{g_{\mathrm{N}}}^{\alpha}(q) \dot{q}=J_{g_{\mathrm{N}}}^{\alpha}(q) T(q) v, \text { if } g_{\mathrm{N}}^{\alpha}(q)=0, \quad \alpha \in \mathcal{I} .
$$

Again, the force applied to the body is given by $\left(J_{g_{\mathrm{N}}}^{\alpha}(q) T(q)\right)^{\top} \lambda_{\mathrm{N}}^{\alpha}$. Nevertheless, there is no reason that $\lambda_{\mathrm{N}}^{\alpha}=r_{\mathrm{N}}^{\alpha}$ and $u_{\mathrm{N}}=J_{g_{\mathrm{N}}}^{\alpha}(q) T(q) v$ if the function $g_{n}$ is not chosen as the signed distance (the gap function). This is the reason why we prefer define directly the normal and the tangential local relative velocities with respect to the twist vector as

$$
u_{\mathrm{N}}^{\alpha}:=G_{\mathrm{N}}^{\alpha}(q) v, \quad u_{\mathrm{T}}^{\alpha}:=G_{\mathrm{T}}^{\alpha}(q) v, \quad \alpha \in \mathcal{I},
$$

and the associated forces as $G_{\mathrm{N}}^{\alpha, \top}(q) r_{\mathrm{N}}^{\alpha}$ and $G_{\mathrm{T}}^{\alpha, \top}(q) r_{\mathrm{T}}^{\alpha}$. For the sake of simplicity, we use the notation $u^{\alpha}:=$ $G^{\alpha}(q) v$ and its associated total force generated by the contact $\alpha$ as $G^{\alpha, \top}(q) r^{\alpha}:=G_{\mathrm{N}}^{\alpha, \top}(q) r_{\mathrm{N}}^{\alpha}+G_{\mathrm{T}}^{\alpha, \top}(q) r_{\mathrm{T}}^{\alpha}$. 
The complete system of equation of motion can finally be written as

$$
\left\{\begin{array}{ll}
\dot{q}=T(q) v, & \\
M \dot{v}=F(t, q, v)+H^{\top}(q) \lambda+G^{\top}(q) r, & \alpha \in \mathcal{E} \\
H^{\alpha}(q) v=0, & \text { if } g_{\mathrm{N}}^{\alpha}(q)>0, \\
r^{\alpha}=0, & \text { if } g_{\mathrm{N}}^{\alpha}(q)=0, \\
K^{\alpha, *} \ni \widehat{u}^{\alpha} \perp r^{\alpha} \in K^{\alpha} & \\
u_{\mathrm{N}}^{\alpha,+}=-e_{r}^{\alpha} u_{\mathrm{N}}^{\alpha,-}, & \text { if } g_{\mathrm{N}}^{\alpha}(q)=0 \text { and } u_{\mathrm{N}}^{\alpha,-} \leqslant 0
\end{array}\right\} \alpha \in \mathcal{I},
$$

where the definition of the variables $\lambda \in \mathbb{R}^{m_{e}}, r \in \mathbb{R}^{3 m_{i}}$ and the operators $H, G$ are extended to collect all the variables for each constraints.

Note that all the constraints are written at the velocity level. In the differential algebraic equations language, we use an index-2 formulation for its ability to design robust time-integrators. Another strong advantage is the straightforward introduction of the contact dissipation processes that are naturally written at the velocity level such as the Newton impact law and the Coulomb friction. Indeed, in Mechanics, dissipation processes are always given in terms of rates of changes, or if we prefer, in terms of velocities.

\subsection{The numerical integration method}

The simulation of systems with clearances needs the application of robust time-stepping schemes with respect to a possible high number of events (impacts, take-off, transitions from sliding to sticking, or vice-versa). This motivates the use of event-capturing schemes where the events are not accurately detected in time, but captured within a time-step. Our scheme will mainly rely on the Moreau-Jean algorithm [35, 48, 49,34,50], where the constraints are solved at the velocity level as it is formulated in (19). It is well-know that velocity-level treatment of the constraints yields violations of constraints, also termed as the drift from the constraints manifold. When we simulate mechanisms with small clearances, this is not tolerable since we have to keep the violation as small as possible with respect to the characteristic length of the clearances. Following [2], we introduce a stabilized index-2 formulation based on the Gear-Gupta-Leimkuhler (GGL) method:

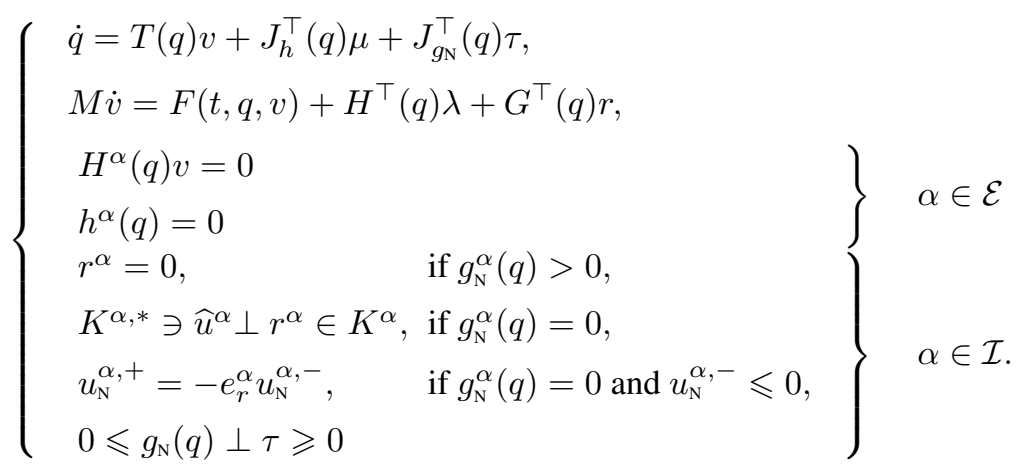

The multipliers $\tau \in \mathbb{R}^{m_{e}}$ and $\mu \in \mathbb{R}^{m_{i}}$ are the multipliers that enforce the constraints $h^{\alpha}(q)=0, \alpha \in \mathcal{I}$ and $g_{\mathrm{N}}^{\alpha}(q)=0, \alpha \in \mathcal{E}$. It is possible to show that the contribution of these multipliers to the first equation vanishes, i.e. $J_{h}^{\top}(q) \mu+J_{g_{\mathrm{N}}^{\top}}^{\top}(q) \tau=0$. After a time-discretization, these terms do not vanish and ensure both the constraints at the velocity level and at the position level.

The discretization of 20 is not straightforward since a direct application of the GGL approach yields spurious oscillations at contact [61]. The oscillations are mainly the result of a bad interaction between the energy injected by the projection and the impact law that depends on the activation of the constraints (see [2] for details). To avoid to project onto a unilateral constraint without applying the impact law, the activation of constraints is made in an iterative way. As in the Moreau-Jean scheme, the primary unknowns are the velocities and the impulses to keep the consistency of the scheme when an impact occurs. Starting from an empty set of active constraints $\mathcal{I}^{0}=\emptyset$, the combined projection scheme is based on two stages at each time-step $k$ of length $h$ : 
1. The projection stage solves the discretized problem for a given set $\mathcal{I}^{\nu}$ of active constraints

$$
\left\{\begin{array}{l}
q_{k+1}=q_{k}+h T\left(q_{k+\theta}\right) v_{k+\theta}+J_{h}^{\top}\left(q_{k+1}\right) \gamma_{k+1}+J_{g_{\mathrm{N}}}^{\top}\left(q_{k+1}\right) \delta_{k+1}, \\
M\left(v_{k+1}-v_{k}\right)-h F_{k+\theta}=H^{\top}\left(q_{k+1}\right) Q_{k+1}+G^{\top}\left(q_{k+1}\right) P_{k+1}, \\
H^{\alpha}\left(q_{k+1}\right) v_{k+1}=0 \\
h^{\alpha}\left(q_{k+1}\right)=0 \\
P_{k+1}^{\alpha}=0, \delta_{k+1}^{\alpha}=0, \\
K^{\alpha, *} \ni \widehat{u}_{k+1}^{\alpha} \perp P_{k+1}^{\alpha} \in K^{\alpha} \\
g_{\mathrm{N}, k+1}^{\alpha}=0, \delta_{k+1}^{\alpha}, \text { if } P_{\mathrm{N}, k+1}^{\alpha}>0, \\
0 \leqslant g_{\mathrm{N}, k+1}^{\alpha} \perp \delta_{k+1}^{\alpha} \geqslant 0 \text { otherwise }
\end{array}\right\} \alpha \in \mathcal{E}
$$

The notation $x_{k+\theta}=(1-\theta) x_{k}+\theta x_{k+1}$ is used for $\theta \in[0,1]$. The term $F_{k+\theta}$ is an approximation of the forces $F(\cdot)$ using a $\theta$-method. The variables $Q_{k+1}$ and $P_{k+1}$ are the approximations of the impulses generated by $\lambda$ and $r$. Compared to the standard Moreau-Jean scheme, the multipliers $\gamma_{k+1}$ and $\delta_{k+1}$ are added to cancel the violations of constraints at he velocity level. They approximate to the impulses generated by the multipliers $\mu$ and $\tau$.

2. The activation stage updates the index set of active constraints $\mathcal{I}^{\nu}$ as follows

$$
\mathcal{I}^{\nu+1}=\mathcal{I}^{\nu} \cup\left\{\alpha \in \mathcal{I} \mid g_{\mathrm{N}, k+1}^{\alpha} \leqslant 0\right\} .
$$

The stages 1 and 2 are iterated until the index set $\mathcal{I}^{\nu}$ is constant. Other variants of the approach based on the nonsmooth generalized- $\alpha$ scheme can be found in [12].

Solving (21) amounts to solving a nonlinear mixed second-order cone problem. We usually perform a Newton linearization of the nonlinear implicit terms in the dynamics, and we solve the resulting linearized mixed secondorder cone problem by a projection/splitting techniques, namely a projected Gauss-Seidel method [4, §13.7.4].

The computation of the gap functions $g_{\mathrm{N}}$ and the local frames at contact $\left(\mathrm{N}, \mathrm{T}_{1}, \mathrm{~T}_{2}\right)$ is of utmost importance in the efficiency of the algorithm. For standard configurations, the gap distances can be explicitly written in closed forms [47]. In industrial applications, when the geometry of the parts are defined by CAD files, it becomes difficult to get some closed form expression of the gap. In our application, we rely on the minimization of the distance between surfaces that are represented by boundary representation (B-rep). Some details are given in Section 3.2.

\section{The C-60 miniature circuit breaker mechanism}

The construction of a miniature circuit breaker as in Figure 1 is simple, however very precise. In fact, a miniature circuit breaker has no replacement parts. It is not designed to be maintained. When a unit goes bad, it is simply replaced. A typical miniature circuit breaker has five main components such as: frame, operating mechanism, electrical contacts, trip unit and arc interrupter.

1. Frame: The frame is composed of high thermal resistant plastic molded case and cover (see Figure 1 a)a). Its primary function is to provide a rigid, mechanically strong, insulated housing in which all the circuit breaker components are mounted and kept in place.

2. Operating mechanism: The operating mechanism mainly consists of seven parts, namely handle, rod, plate, hook, tripping bar, moving contact and needle (see Figure 1 b)-(c)). It provides the means of opening and closing the circuit. It has a three-position switch ("ON," "OFF," and "TRIP"). In the event of electrical fault such as an over-current or abnormal conditions in the electrical network, one of the functions of the circuit breaker is to safeguard the electrical network by breaking the circuit using TRIP operation. The power supply can be cut from the source by manually switching OFF the breaker or due to the TRIP operation. The position of the handle of the breaker makes it easy for a maintenance person to determine why power has been cut. 
3. Electrical contacts: The flow of the current in an electrical circuit is controlled by a circuit breaker through the circuit breaker's contacts (see Figure 1 b b)-(c)). One of the contacts is mounted on the frame, and is known as the fixed contact. The other contact becomes part of the operating mechanism, and is called the moving contact. When a circuit breaker is turned off or is tripped by a fault current, the circuit breaker interrupts the flow of the current by separating its electrical contacts.

4. Trip unit: The trip unit is the brain of the miniature circuit breaker. It activates the operating mechanism in the event of a prolonged overload or short circuit. This type of circuit breaker uses a thermal magnetic mechanism. This is the predominant trip unit technology used in the domestic market. A bi-metal and an electromagnet work together to provide overload and short-circuit protection.

5. Arc interrupter: When the circuit breaker contacts open, current continues to flow for a short time by arcing across the air space between the moving and fixed contact. The function of the arc interrupter is to divide and cool the arc. It prevents the arc to sustain for longer time. In the miniature circuit breaker, the arc chute method is used to break the arc into smaller pieces.

Mechanism working principle: All the mechanism parts are enclosed in-between the case and cover parts (see Figure 1(a) and Figure 10(b)). These parts are connected to each other through a revolute joint or frictional contact. In the following section we will see the detailed description of these joints and contacts. In the first step, the primary function of a mechanism is usually formulated in terms of kinematical quantities (link geometry, kinematic constraints, etc). Also the various geometrical relations resulting from the kinematical analysis of the linkage mechanism are an essential ingredient for the dynamic analysis. The kinematical analysis of a miniature circuit breaker mechanism (hereafter called the C-60 mechanism) is of great importance. The C-60 mechanism consists of seven links, seven revolute joints and four frictional contacts (see Figure 4(a)). It has forty two degrees of freedom. The operating mechanism provides three key positions (ON/OFF/TRIP) which are explained as follows:

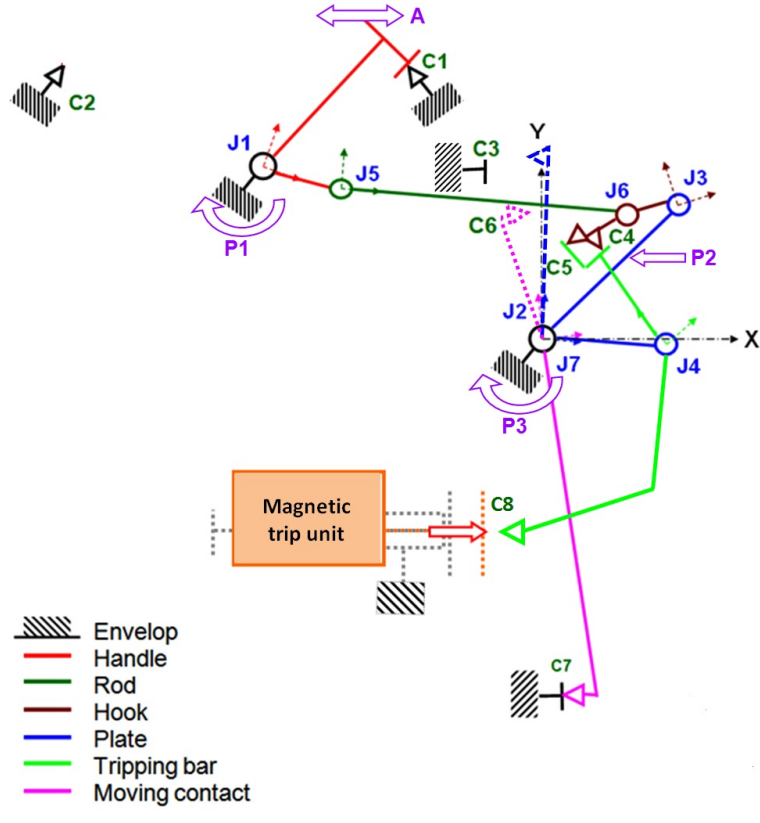

(a) $\mathrm{ON}$ (close) position

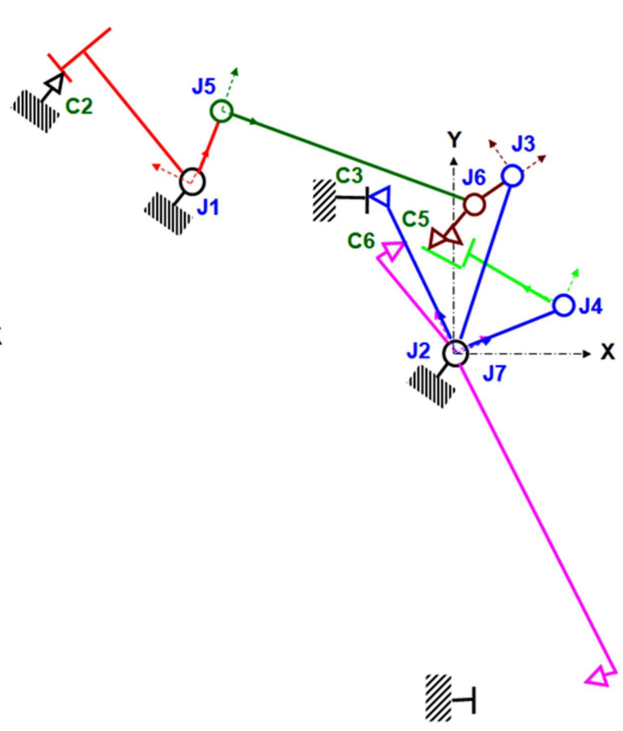

(b) OFF (trip or open) position.

Fig. 4: Kinematic representations of the C-60 mechanism.

1. The close operation leads to ON position of the breaker. In close operation (see Figure $4(\mathrm{a})$ ), the operating handle $(A)$ is rotated clockwise which closes the contacts $C_{5}$ and $C_{4}$ through the revolute joints $J_{1}, J_{5}, J_{6}, J_{3}$ and $J_{4}$. The frictional contacts $C_{5}$ and $C_{4}$ have a specific wedge shape profile, which enables the locking between 
the hook and tripping bar. After the activation of the contacts $C_{5}$ and $C_{4}$ the motion has been transferred to the moving contact through the plate by revolute joints $J_{2}$ and $J_{7}$, which ensure closing of the contact between the moving and the fixed contacts. During close operation the handle spring $\left(P_{1}\right)$ and the mechanism springs $\left(P_{2}\right.$ and $P_{3}$ ) get charged, which will be used for the trip operation of the breaker.

2. The trip operation leads to TRIP position of the breaker. In the trip operation (see Figure $4(\mathrm{~b})$ ), the activation of the tripping coi ${ }^{5}$ causes the tripping plunger ${ }^{6}$ to be attracted to close the contact $C_{8}$, thus releasing the contact $C_{7}$ through $C_{4}$ and $C_{5}$. In response, the handle and mechanism spring discharge by moving the handle and plate to the open position.

3. The open operation results in switching OFF the breaker. The function of open operation is the same as the trip operation, to break the circuit (i.e. to release the contact $C_{7}$ ), and it may be necessary for maintenance of the electrical network. However this operation is not automated: the operator has to rotate the handle in the counter clockwise direction. The contact $C_{7}$ between the moving and the fixed contact is opened through the revolute joints $J_{1}, J_{5}, J_{6}, J_{3}, J_{4}, J_{2}$ and $J_{7}$.

3.1 Modeling of the spatial revolute joint with clearances

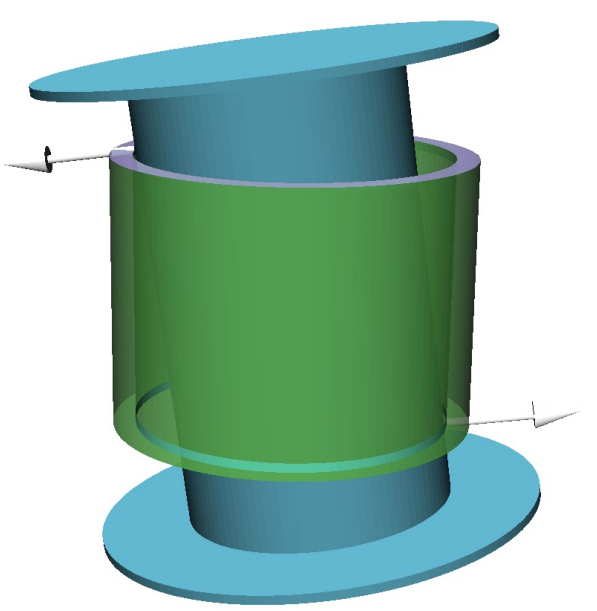

(a) Cylinder/Cylinder contact with axial mis-alignment

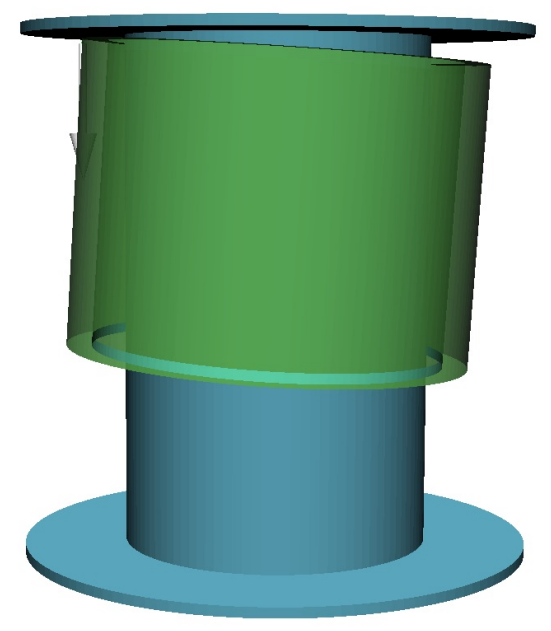

(b) Cylinder/plane contact for contact with flanges.

Fig. 5: Two kinds of contacts in spatial revolute joint with clearances showing contact forces in SICONOS.

The modeling of spatial revolute joints with clearances is illustrated in Figure 5 for a simple case of a journal with a constant radius $r_{j}$ with flanges and a bearing of constant radius $r_{b}$ (see also Figure 7). As illustrated in Figures 5 and 3.1. the contact mode between the two parts may be enumerated and leads to configurations from one to four contacts points, or to line and plane contact surfaces. For such standard configurations, it is possible to write closed-form formulations of the gap functions and local frames at contact. Nevertheless, it does not take into account possible varying journal and bearing radius and chamfers that are commonly used in mechanical design. In order to take into account complex industrial geometries, we prefer to rely on the computation of distances and local frames given by CAD software that uses B-rep representation. Once the pairs of two contacting surfaces are defined, we use optimization techniques to compute the minimal distance between two parametric surfaces described by splines or NURBS (see Section 3.2 for details).

When we use a B-rep surfaces and some minimization technique, we assume that locally the optimization problem is convex and yields a unique solution. Two drawbacks are implied : a) we generate just one contact point for

5 A control device that utilizes a solenoid to open a circuit breaker.

6 Under normal working condition, the plunger is held in a position by spring because the magnetic field generated by the coil is not sufficient to release the latch. When a fault current flows, the magnetic field generated by the coil is sufficient to overcome the spring force holding the plunger in position. And hence the plunger moves and then actuates the tripping mechanism. 


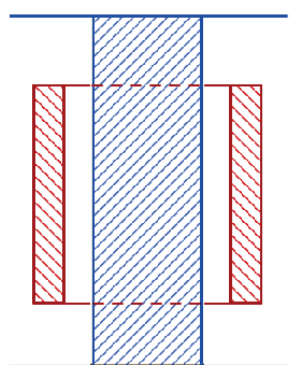

(a) No contact.

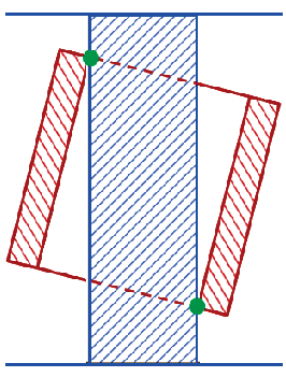

(e) Two point contact with journal.

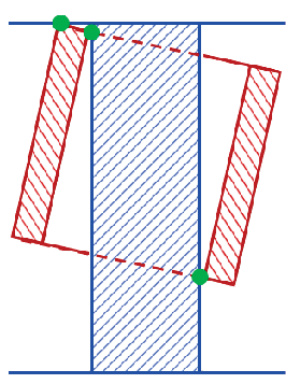

(i) Three point contact with journal and flange.

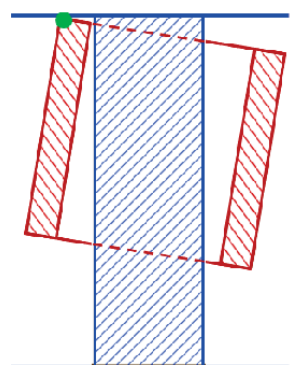

(b) One point contact with flange.

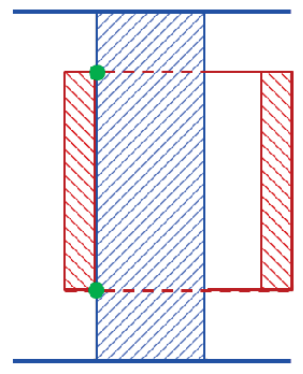

(f) Line contact.

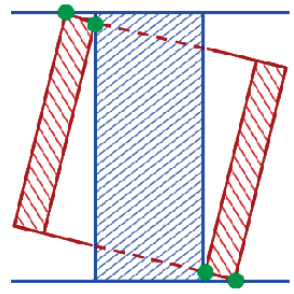

(j) Four point contact with journal and flange.

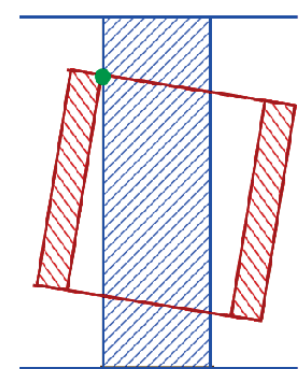

(c) One point contact with journal.

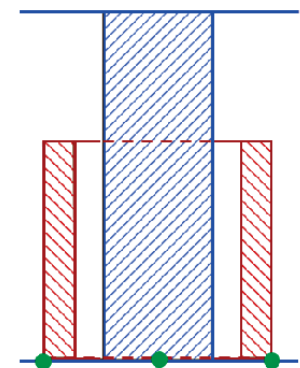

(g) Plane contact with flange.

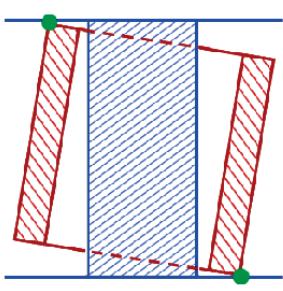

(k) Two point contact with flange.

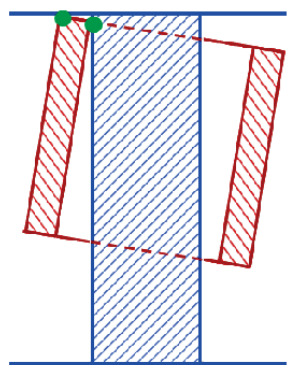

(d) Two point contact with journal and flange.

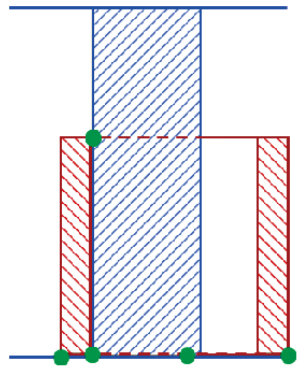

(h) Line and plane contact with journal and flange.

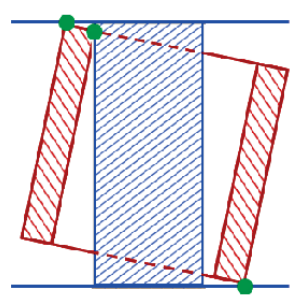

(l) Three point contact with journal and flange.

Fig. 6: Two kinds of contacts in spatial revolute joints with clearances

the line and plane contact and b) the minimum may be a local one if the problem is not convex. It is clear that to transfer the correct torque generated by a line (resp. plane) contact we need at least 2 (resp. 3) contact points. Furthermore, the contact points must be located in such a way that the pivoting of the parts around a leaving contact point is consistent. For this reason, we prefer to specify and to split the contact surfaces in order to perform an explicit location of the contact points. If the surface is not convex, we perform a convex decomposition of the 
surfaces. If the surface is convex, but not strictly convex (plane or line contacts), we split the contacting surfaces as in the examples that follow.

Modeling of cylinder/cylinder contact. For dealing with the cylinder/cylinder contact with possibly line contact, the ideal revolute joint is replaced by a journal and two circular rings at the extreme ends of the bearing (see Figure 4), which acts as a spatial revolute joint with clearance. The axial and radial clearances in the revolute joint are modeled by introducing six degrees of freedom between the bearing and the journal. The addition of degrees of freedom allows to take into account the so-called polarization effect in Figure 7(c) that corresponds to the out-of-plane motion of the parts.

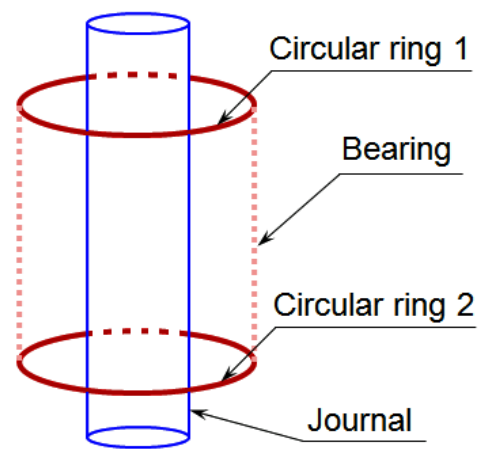

(a) Gap distance are computed between the circular rings (in red) and the journal cylinder (in blue)

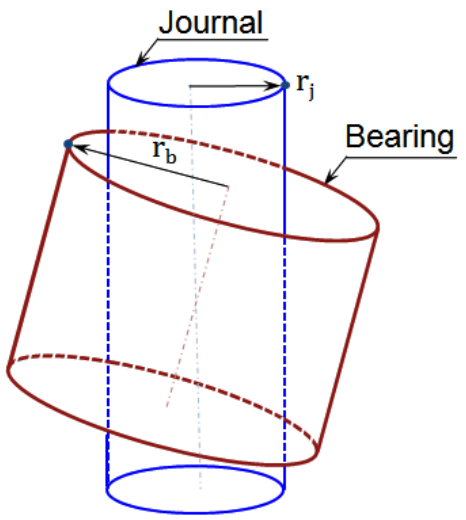

(b) Axial mis-alignment

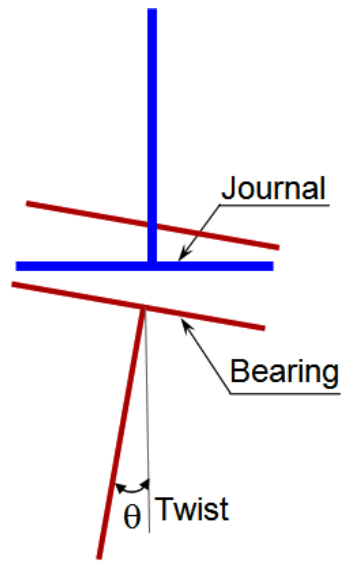

(c) Polarization effect: out-ofplane motion of the mechanism due to clearances.

Fig. 7: Generic representation of a 3D revolute joint with clearance : cylinder/cylinder contact

Modeling of cylinder/plane contact. For dealing with the contact with the flanges, the modeling of the cylinder/cylinder contact between the journal and the bearing has to be completed to take into account the fact that the relative motion between them is restricted by the internal surface of the bearing and the flanges of the journal. The contact between the flange and the bearing top/bottom surface is a plane-plane contact (see $(\mathrm{g})$ and $(\mathrm{h}))$.

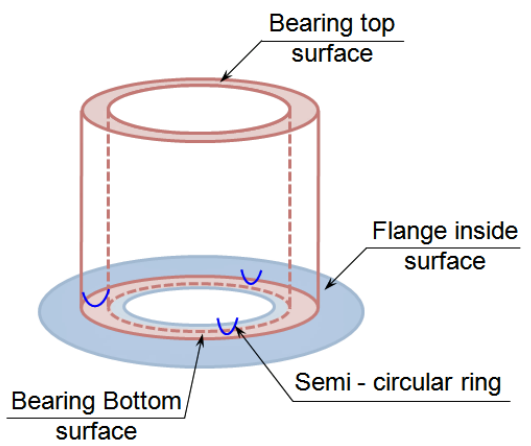

Fig. 8: Modelling of plane-plane contact between the bearing and the journal flanges or plane stops.

In the numerical practice with rigid bodies, the plane-plane contact is completely described by three contact points. However, as we said before, the limitations of the plane-plane contact through a minimization procedure of the contact distance are : a) the location of the three contact points is under-determined and the contact detection algorithm may randomly choose a location and b) this can lead to a simulation extra-cost as the contact detection is done on the entire area. The random location of the contact point can affect the frictional behavior of the contact. 
This is the price to pay with a rigid modeling of the parts and a reduced space discretization of the contact surface. In order to control the location of contact points, enabling parametric studies of the influence of the contact location, we decided to adopt a dedicated modeling of plane-plane contact illustrated on the Figure 8 The contact between the flange and the bearing face is modeled by considering the plane surface of the flange, while the plane surface of the bearing is replaced by three semi-circular equidistant rings (see Figure 8). This methodology allows us to perfectly constrain the plane-plane contact with a significant reduction in the simulation time.

Let us remark that in manufactured products, an ideal plane-plane contact is impossible due to the presence of surface roughness and waviness. Our methodology choose the location of contact points. A step forward could be to perform a sensitivity analysis to the locations of the contact points in the plane. In our case, this location has been checked such that it reproduces the experimental behavior of the mechanism.

Modeling of cylinder/plane contact. To tackle this situation we consider the combination of plane surface and two semi-circular rings on each of the plane contacting surface (see Figure 9/a)-(b)). It will allow us to simulate the twisted contact surfaces and ensure a minimum quantity of three contact points.

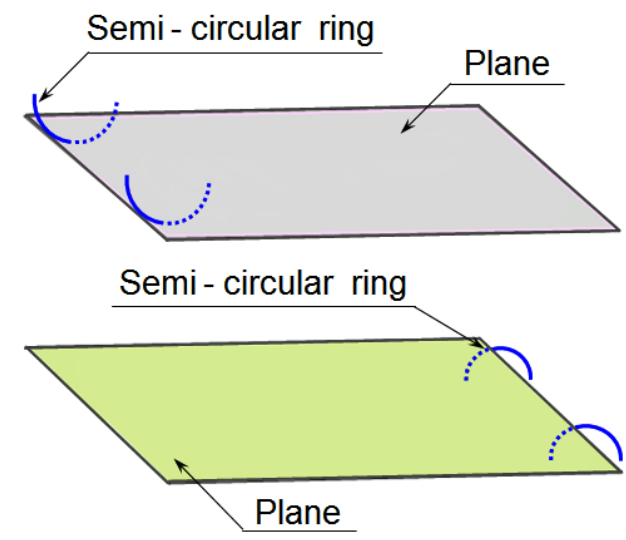

(a) Plane surface and two semi-circular rings for the first plane surface.

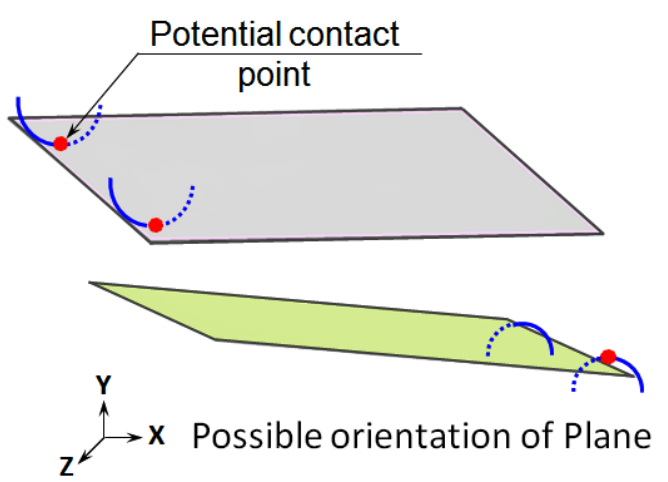

(b) Plane surface and two semi-circular rings for the second plane surface.

Fig. 9: Strategy to model the plane-plane contact.

Illustration of the spatial revolute joint $J_{1}$ between the case, the cover and the handle Let us detail the modeling of the spatial revolute joint with axial and radial clearances on the example of the the revolute joint $J_{1}$ between the case, the cover and the handle illustrated in Figure 10. The protrusion on the case and cover acts as a journal and the cavity on the handle acts as a bearing. The highlighted blue surface of the case, the cover and the handle constitute a revolute joint with clearance. The highlighted red surface on the case and the cover acts as a flange and the axial motion of handle is restricted by the case and the cover (see Figure 10 .

Remark 1 Modelling 3D revolute or prismatic joints is a particularly difficult task, because of cylinder/bore or plane/plane contacts, which may yield conformal contacts. Various contact models may yield quite different outcomes [54,55]. The simplified models we choose in this study, encapsulate essential features of the considered joints, as the results of section 5 show.

\subsection{Contact offset and contact detection in SiCONOS/MECHANICS.}

The modeling and the simulation in this article are performed with SICONOSand its SiCONOS/MECHANICS module. SICONOSis a generic software code for the simulation of nonsmooth dynamical system, and especially systems with contact, friction and impacts [7,3]. SiCONOSis an open-source software under Apache 2.0 license and it is freely available. The SiCONOS/MECHANiCs software allows the user to instantiate a collection of bodies described by the Newton/Euler equations linked with ideal joints and frictional contact interface. Practically, the 

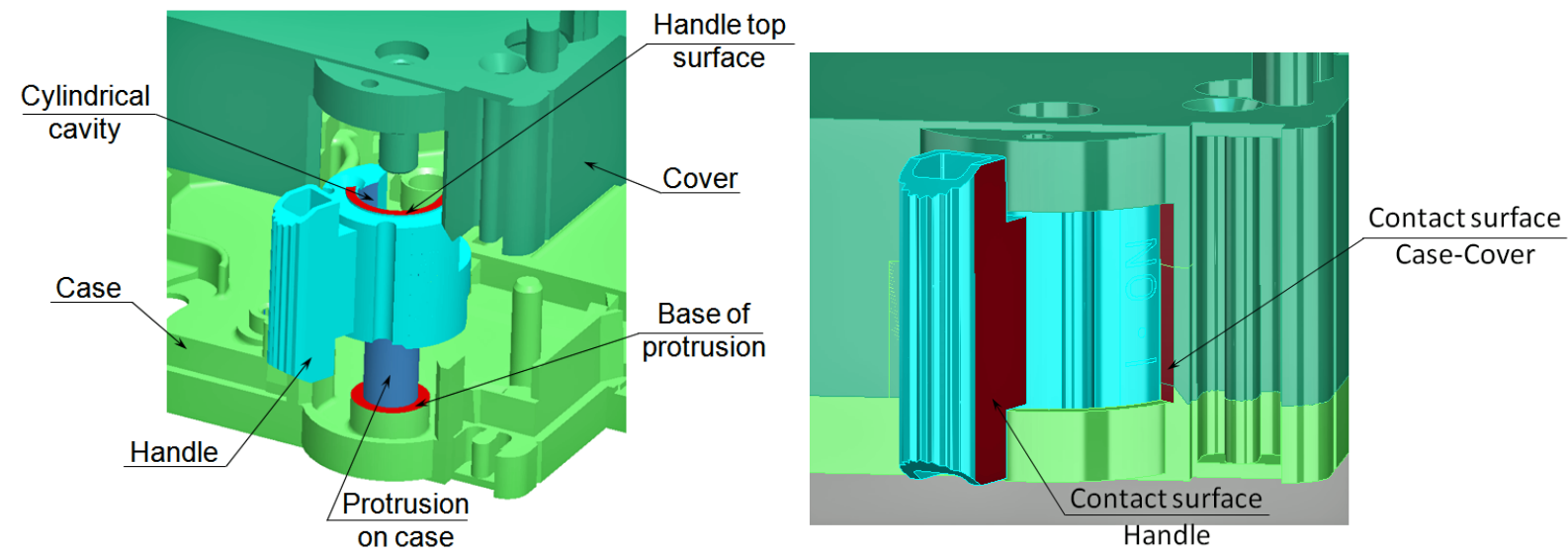

Contact
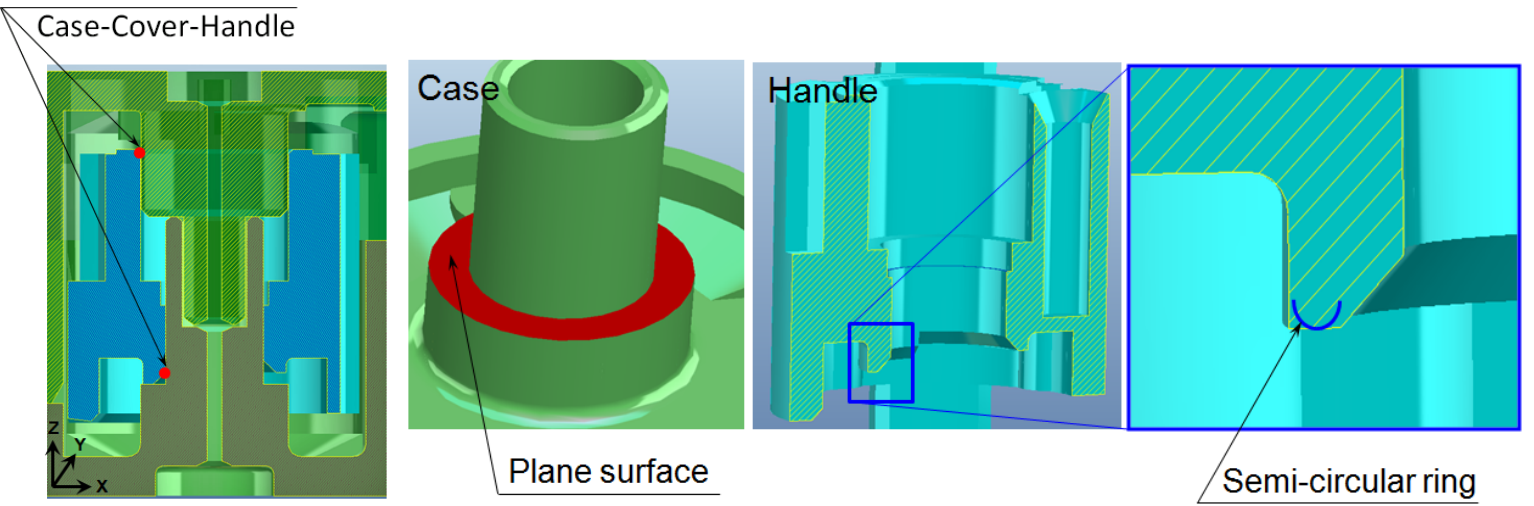

Fig. 10: Revolute joint with clearance $J_{1}$ between case, handle and cover.

software uses geometrical representations based on boundary representation (B-rep) with the industrial CAD library OPENCASCADE [17]. In our examples, we use also its Python wrapper PYTHONOCC [56] for manipulation and visualization.

For the contact description and detection, a virtual boundary is constructed around the real CAD geometry based on Minkowsky sum with a given contact offset. Once the minimization process to compute the gap between parts is done, we subtract the contact offset from the normal gap. Usually the contact offset values, denoted by $g_{o}$, are of the order $10^{-5}$ to $10^{-6} \mathrm{~mm}$. The contact offset is very useful to avoid penetration between the real CAD geometries (see Figure 11) in order to simplify the contact distance computation. In case of the revolute joint, we also use the contact offset to modify the contact clearances without regenerating the CAD files. The CAD files are designed with a maximum allowed clearances. The distance obtained by the mimimization process is then modified by subtracting a contact offset that defined the final clearance. The process amounts to virtually increasing or decreasing the radius of the cylinders. The contact offset is a user parameter that can be defined as a variable in Siconos/MECHANICS module. It allows us to adapt the value of the radial clearance in the joint and the simulations can be performed without updating the CAD geometry of the contacting bodies.

In case of contact offset equal to the distance between the surfaces defined in the CAD files, the revolute joint becomes perfect because the contact distance vanishes. As the value of contact offset increases from 0 to the maximal distance between the surfaces defined in the CAD files, the radial clearance in the joint decreases.

The collision detection is a geometric contact determination problem that depends on the spatial relationship of the objects, while the collision response is a dynamical problem. In short, the accuracy in the dynamic response depends on the collision detection. Therefore, the collision detection is an important step for a good prediction of the behaviour of multibody systems. The contact detection process is generally based on a) a broad phase, where a collection of pairs of geometric objects (simple primitive or simple B-Rep instances) are detected within a given 


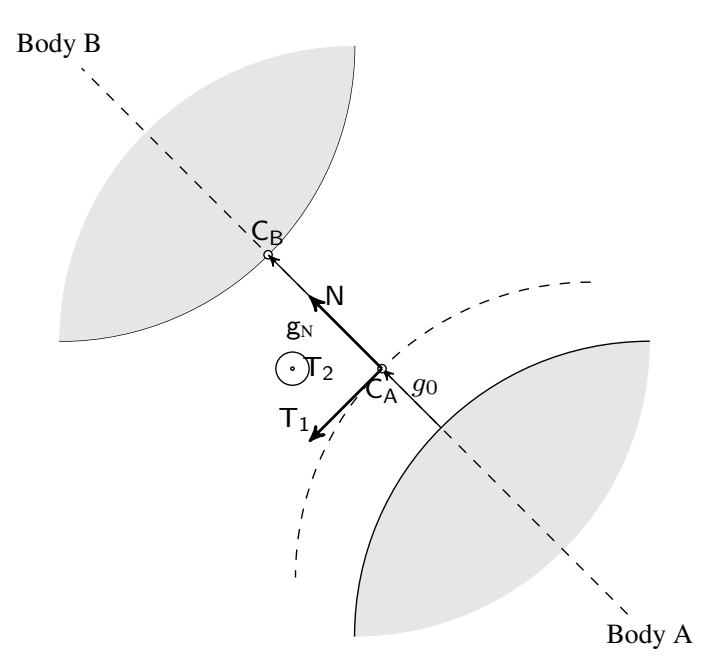

Fig. 11: Contact local frame with contact offset $g_{0}$.

coarse distance, and b) a narrow phase, where the computation of distances and local frames at contact is made. When we deal with mechanisms, the broad phase of the contact detection is skipped in SICONOS/MECHANICS. Instead, the user provides the pairs of primitive or B-Rep objects that will potentially be in contact by directly providing the B-rep files of the contacting objects. These files may be different from the files that define the parts. This manner renders possible fine tuning of the contact discretization, that is not possible with an algorithmic broad phase contact detection. Furthermore, the contact detection is really efficient and robust. After this step, we minimize the distance between two B-rep objects assuming that the problem is convex. If not, the user must precise the contact surfaces by operating some partition of complicated surfaces or keep the point given by the local minima. The minimization algorithm is N2QN1 [43,44] developed by INRIA. It is designed to minimize functions $f(x)$ depending on a small or medium number of variables $x$ subject to bound constraints $(a \leqslant x \leqslant b)$. They implement a quasi-Newton (BFGS) technique with line-search.

\section{Sensitivity analysis}

In order to ensure the robust performance of the mechanism, it is necessary to simulate and study the effect of dimensional variations in the manufacturing process due to the allocated tolerances on the parts in an assembly. Most often the manufacturing and assembly of the parts in the product happen in batches. The batch production is more prone to the random variations in the joint clearances in the mechanism assembly. Our aim is to predict well in advance during the product design stage the variations in the overall performance of the product due to the uncertainty in the joint clearances. The statistical tolerance analysis is a powerful and most economical method to forecast the output variations due to the uncertainty associated with the assembly process [18]. There are different

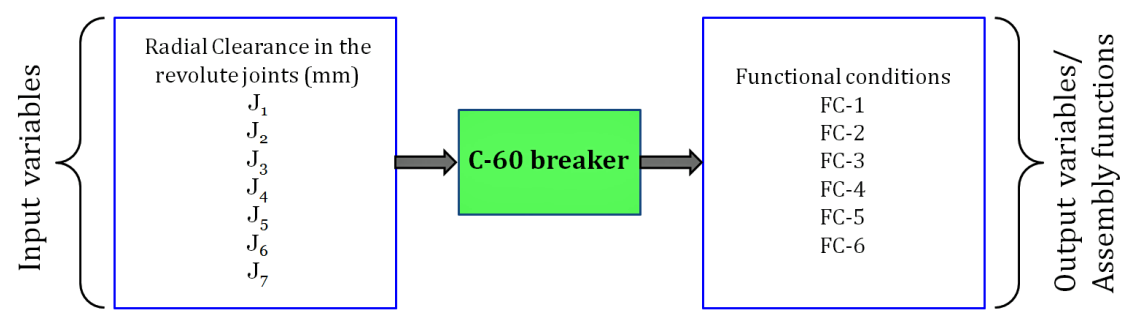

Fig. 12: Variables in the statistical analysis.

methods of statistical tolerance analysis: Root Sum of Squares (RSS), extended Taylor series, quadrature technique and Monte Carlo simulation [18,51]. In our case, the input variables and the functional conditions are illustrated in 
Figure 12 and described in Table 1] The first three methods are used when the assembly function can be expressed explicitly as an input variables of each assembled parts. The Monte Carlo method can be used very efficiently in the situation where the assembly function/output variables is not available, or impossible to write explicitly [64]. In order to allocate the tolerances on the parts, the manufacturing cost and the quality of the parts are the important aspects. The quality of the parts in terms of geometrical and dimensional variations, plays an important role in the assembly of the product. Wu et. al. [75] proposed the Monte Carlo simulation and genetic algorithm methodology to minimize the manufacturing cost and to ensure the assembly requirements.

In the $3 \mathrm{D}$ analysis, it is very difficult to write the different assembly functions (functional conditions) explicitly or implicitly. Here the aim is to find out the influence of the joint radial clearance in 3D on the functional conditions of the C-60 breaker. To this aim we used the Monte Carlo method to simulate the geometrical variations of the C-60 breaker mechanism.

\subsection{Monte Carlo simulation}

The flow chart of the Monte Carlo simulation is depicted in Figure[13. It is really similar to the one in [18]. Random

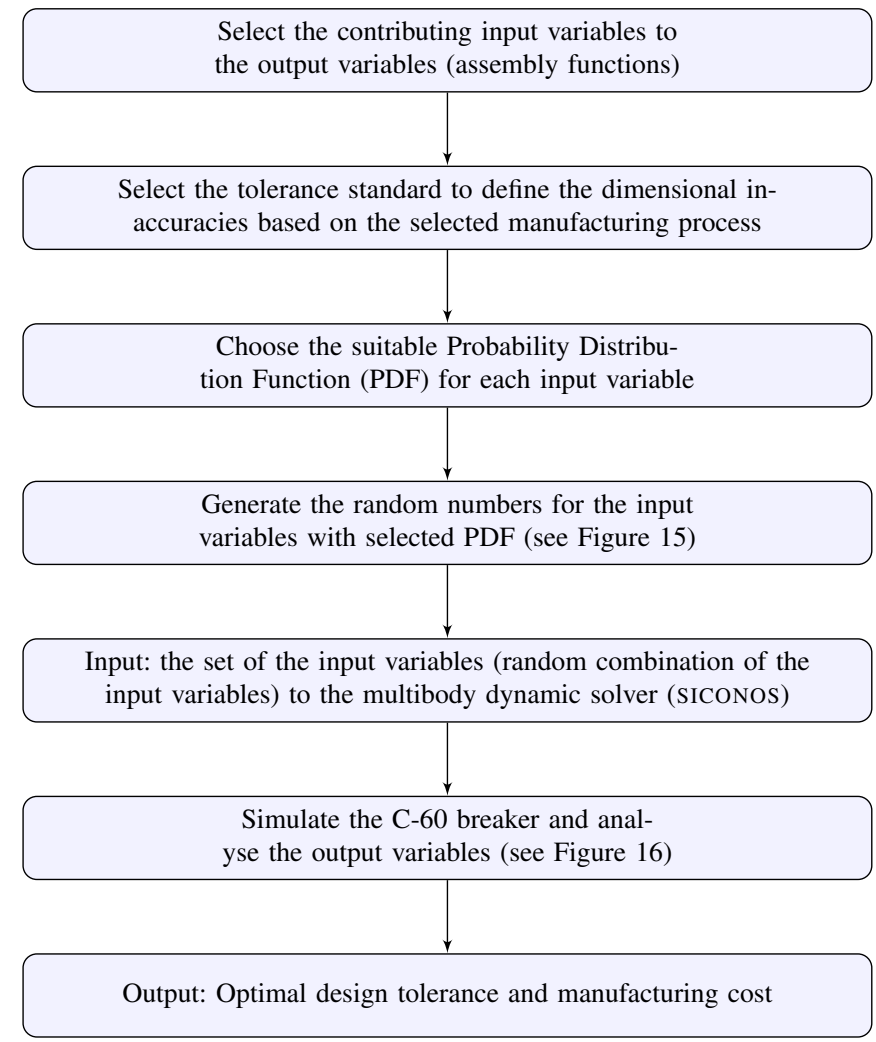

Fig. 13: Monte Carlo simulation flow chart.

values for each part (component) in the assembly are generated according to the probability distributions (uniform, normal, Poisson, Weibull, triangular, geometric, exponential, etc.) used for the manufacturing process [59]. Generally, for the plastic molded parts, the normal distribution is widely used, so we chose it for our case study of C-60 circuit breaker, see Figure 14. In the case of C-60 breaker, the nominal value and the tolerances on the dimensions of the parts associated with joints $J_{1}, \ldots, J_{7}$ vary from $0.01 \mathrm{~mm}$ to $0.13 \mathrm{~mm}$. As per the rule of thumb, minimum $0.01 \mathrm{~mm}$ clearance is required to have a relative motion between the parts joined by revolute joint. For the normal distribution we need the mean value $\bar{m}$ of the tolerance band and the standard deviation $\sigma$ to generate the spread. As depicted in Figure 15 the spread is bounded by the USL and LSL values. In the further step, the random combination of the generated random input variables is generated. This set of the seven random input variables is the input to the multibody dynamic solver SICONOS. The values of the output variables (assembly function/functional 


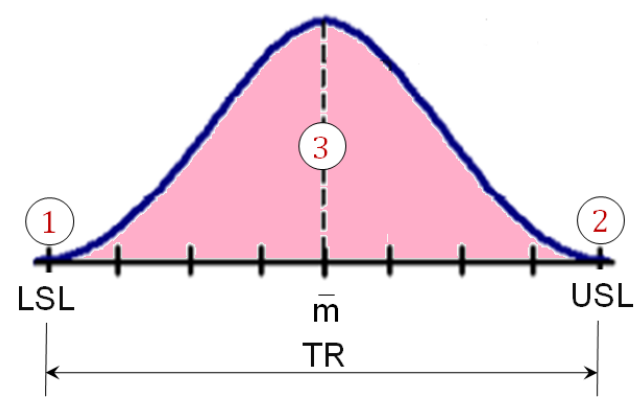

Fig. 14: Methodology adopted for the worst-case and Monte Carlo simulation.

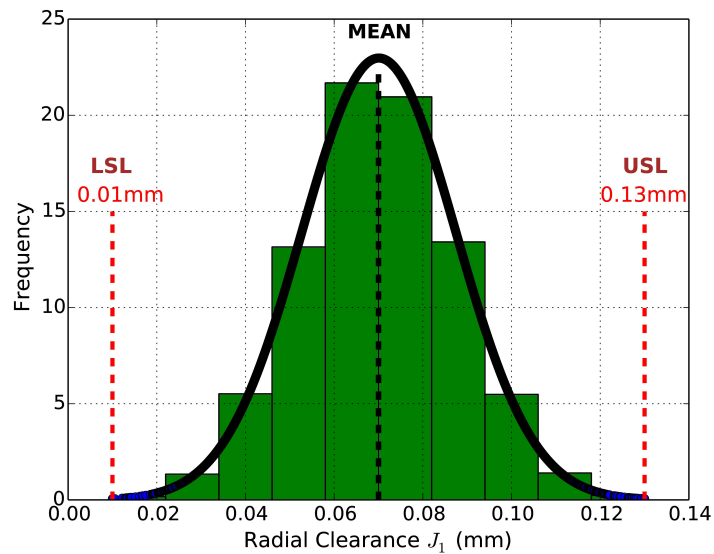

(a) Joints $J_{1}, \ldots, J_{7}, \bar{m}=0.07 \mathrm{~mm}, \sigma=0.0175$.

Fig. 15: Generated random numbers for the joints $J_{1}, J_{2}, J_{3}, J_{4}, J_{5}, J_{6}$ and $J_{7}$.

conditions) are computed for each set of the input variables. In this case, input variables are in the form of radial clearance for each revolute joint. The output variables (assembly function/functional conditions) are given in Table 1 These output variables are functions of the input variables (radial clearance in the joints),

Table 1: Output variables of the C-60 breaker.

\begin{tabular}{|c|l|}
\hline FC - Name & \multicolumn{1}{|c|}{ Description of the Functional Conditions (FC) } \\
\hline FC - 1 & Contact Force (N) \\
\hline FC - 2 & Distance between Needle - Tripping bar pin position in X direction (mm) \\
\hline FC - 3 & Distance between Needle - Tripping bar pin position in Y direction (mm) \\
\hline FC - 4 & Distance between Needle - Lamage in X direction (mm) \\
\hline FC - 5 & Distance between Needle - Lamage in Y direction (mm) \\
\hline FC - 6 & Distance between Tripping bar - Plunger in X direction (mm) \\
\hline
\end{tabular}

$$
\mathrm{FC}_{k}=f\left(J_{i}\right)
$$

where $k \in\{1,2, \ldots, 6\}$ and $i \in\{1,2, \ldots, 7\}$. A large number of samples of the input variables (radial clearance in the joints) are combined to get a reliable measure of the output variable (assembly function/functional conditions) distribution. 
Table 2: CG position in the local frame.

\begin{tabular}{|c|c|c|c|}
\hline \multirow{2}{*}{$\begin{array}{c}\text { Name of the } \\
\text { part (body) }\end{array}$} & \multicolumn{3}{|c|}{ CG position } \\
\cline { 2 - 4 } & $X(\mathrm{~mm})$ & $Y(\mathrm{~mm})$ & $Z(\mathrm{~mm})$ \\
\hline Handle & 1.1233 & 2.9393 & 4.0018 \\
\hline Rod & 7.5625 & -1.2245 & -0.1586 \\
\hline Hook & -1.9668 & 0.7198 & 1.2825 \\
\hline Plate & 3.3191 & 4.3655 & -4.7064 \\
\hline Moving contact & 7.7912 & -1.7807 & 4.0343 \\
\hline Tripping bar & -1.9723 & -2.3251 & -4.9872 \\
\hline Needle & 0.0000 & 0.0000 & 5.0000 \\
\hline Cover & 2.0751 & 3.2371 & -7.8605 \\
\hline
\end{tabular}

Table 3: Inertial properties of the C-60 mechanism parts (body)

\begin{tabular}{|c|c|c|}
\hline \multirow{2}{*}{$\begin{array}{l}\text { Name of the } \\
\text { part (body) }\end{array}$} & \multirow{2}{*}{$\begin{array}{c}\text { Mass } \\
(\mathrm{kg})\end{array}$} & Inertia matrix of the parts $\left(\mathrm{kg} \mathrm{m}^{2}\right)$ \\
\hline & & $\left(\left(I_{X X}, I_{X Y}, I_{X Z}\right),\left(I_{Y X}, I_{Y Y}, I_{Y Z}\right),\left(I_{Z X}, I_{Z Y}, I_{Z Z}\right)\right)$ \\
\hline Handle & $1.30 \cdot 10^{-03}$ & $\begin{array}{l}\left(\left(3.8912 \cdot 10^{-08},-6.0151 \cdot 10^{-09},-2.8918 \cdot 10^{-10}\right)\right. \\
\left(-6.0151 \cdot 10^{-09}, 2.7959 \cdot 10^{-08},-1.9219 \cdot 10^{-09}\right) \\
\left.\left(-2.8918 \cdot 10^{-10},-1.9219 \cdot 10^{-09}, 4.1931 \cdot 10^{-08}\right)\right)\end{array}$ \\
\hline Rod & $5.00 \cdot 10^{-04}$ & $\begin{array}{c}\left(\left(2.4550 \cdot 10^{-09}, 3.1330 \cdot 10^{-10},-1.4650 \cdot 10^{-09}\right)\right. \\
\left(3.1330 \cdot 10^{-10}, 2.1629 \cdot 10^{-08}, 6.8441 \cdot 10^{-10}\right) \\
\left.\left(-1.4650 \cdot 10^{-09}, 6.8441 \cdot 10^{-10}, 2.0917 \cdot 10^{-08}\right)\right)\end{array}$ \\
\hline Hook & $9.00 \cdot 10^{-05}$ & $\begin{array}{l}\left(\left(3.0507 \cdot 10^{-10}, 8.1781 \cdot 10^{-11},-5.4128 \cdot 10^{-11}\right)\right. \\
\left(8.1781 \cdot 10^{-11}, 4.4192 \cdot 10^{-10}, 1.9009 \cdot 10^{-11}\right) \\
\left.\left(-5.4128 \cdot 10^{-11}, 1.9009 \cdot 10^{-11}, 6.2063 \cdot 10^{-10}\right)\right)\end{array}$ \\
\hline Plate & $1.35 \cdot 10^{-03}$ & $\begin{array}{l}\left(\left(2.9772 \cdot 10^{-08}, 3.5465 \cdot 10^{-09}, 4.7496 \cdot 10^{-10}\right)\right. \\
\left(3.5465 \cdot 10^{-09}, 2.5095 \cdot 10^{-08}, 6.0505 \cdot 10^{-10}\right) \\
\left.\left(4.7496 \cdot 10^{-10}, 6.0505 \cdot 10^{-10}, 3.7698 \cdot 10^{-08}\right)\right)\end{array}$ \\
\hline Moving contact & $2.50 \cdot 10^{-03}$ & $\begin{array}{l}\left(\left(2.3767 \cdot 10^{-08}, 8.5414 \cdot 10^{-10},-1.1814 \cdot 10^{-08}\right)\right. \\
\left(8.5414 \cdot 10^{-10}, 1.7702 \cdot 10^{-07},-1.4361 \cdot 10^{-10}\right) \\
\left.\left(-1.1814 \cdot 10^{-08},-1.4361 \cdot 10^{-10}, 1.8255 \cdot 10^{-07}\right)\right)\end{array}$ \\
\hline Tripping bar & $7.70 \cdot 10^{-04}$ & $\begin{array}{c}\left(\left(1.8429 \cdot 10^{-08},-1.9231 \cdot 10^{-09}, 5.9362 \cdot 10^{-09}\right)\right. \\
\left(-1.9231 \cdot 10^{-09}, 3.1822 \cdot 10^{-08},-5.3010 \cdot 10^{-09}\right) \\
\left.\left(5.9362 \cdot 10^{-09},-5.3010 \cdot 10^{-10}, 2.7636 \cdot 10^{-08}\right)\right)\end{array}$ \\
\hline Needle & $3.9 \cdot 10^{-04}$ & $\begin{array}{l}\left(\left(8.5112 \cdot 10^{-09}, 0,0\right)\right. \\
\left(0,1.9729 \cdot 10^{-10}, 0\right) \\
\left.\left(0,0,8.5611 \cdot 10^{-09}\right)\right)\end{array}$ \\
\hline Cover & $3.69 \cdot 10^{-02}$ & $\begin{array}{c}\left(\left(2.1852 \cdot 10^{-05}, 1.8145 \cdot 10^{-07},-5.5719 \cdot 10^{-07}\right)\right. \\
\left(1.8145 \cdot 10^{-07}, 3.4871 \cdot 10^{-05}, 1.4801 \cdot 10^{-08}\right) \\
\left.\left(-5.5719 \cdot 10^{-07}, 1.4801 \cdot 10^{-08}, 1.3654 \cdot 10^{-05}\right)\right)\end{array}$ \\
\hline
\end{tabular}

Table 4: Contact parameters used in the simulation with clearance in the joints.

\begin{tabular}{ll}
\hline \hline Coefficient of friction metal/plastic contact $\mu$ & 0.1 \\
Coefficient of friction plastic/plastic contact $\mu$ & 0.3 \\
Coefficient of restitution $e_{r}$ & 0.0 \\
Radial clearance interval $c_{r}$ & {$[0.01 \mathrm{~mm}, 0.13 \mathrm{~mm}]$} \\
\hline
\end{tabular}

Table 5: Parameters used in simulation for clearance in the joints.

\begin{tabular}{llll}
\hline \hline Contact detection precision & $1 \cdot 10^{-8}$ & Time step $h$ & $1 \cdot 10^{-3} \mathrm{~s}$ \\
Simulation interval from 0 to $T$ & $7 \mathrm{~s}$ & & \\
\hline
\end{tabular}

\subsection{Numerical results}

The geometric and inertial properties of the C-60 mechanism are given in Tables 2 and 3 . The initial conditions are given in Table 6 and the parameters used for the simulations are given in Tables 4 and 5 . Let us remark that the coefficient of restitution is equal to 0 , which corresponds to perfectly plastic impacts. This is justified by the fact that we have mainly contacts between plastic parts with Teflon ${ }^{\circledR}$ coating. The coefficient of restitution is generally very low for this type of material. We tried other values between 0 and 0.1 but it does not radically change the 
Table 6: Initial position of the parts (bodies)in the inertial frame

\begin{tabular}{|c|c|}
\hline $\begin{array}{c}\text { Name of the } \\
\text { part (body) }\end{array}$ & $(X, Y, Z, q)$ \\
\cline { 2 - 2 } Handle & $(-16.100,10.000,0.500,-0.3229,0,0,1)$ \\
\hline Rod & $(-11.659,8.461,10.300,-0.0956,0,0,1)$ \\
\hline Hook & $(7.7669,7.637,7.100,0.25650,0,1)$ \\
\hline Plate & $(0.0,0.0,9.700,-0.0349,0,0,1)$ \\
\hline Moving contact & $(0.0,0.0,0.0,-1.2437,0,0,1)$ \\
\hline Tripping bar & $(7.000,-0.250,7.100,0.6284,0,0,1)$ \\
\hline Needle & $(0.0,0.0,5.100,1.571,1,0,0)$ \\
\hline Cover & $(0.0,0.0,13.550,-1.571,1,-1,1)$ \\
\hline
\end{tabular}

results. We have carried out 30850 simulations to get the reliable results. The time required for each simulation is approximately $810: 7$ A parallel computing method has been for the simulations. The simulations are performed on the University of Grenoble-Alpes cluster CIMENT ${ }^{8}$ with eight nodes and one hundred sixty CPUs. Monte Carlo simulations can work well with any kind of distributions, only by modifying the random number generator to the desired distribution. It is useful for both linear and nonlinear response functions, because the values of the response

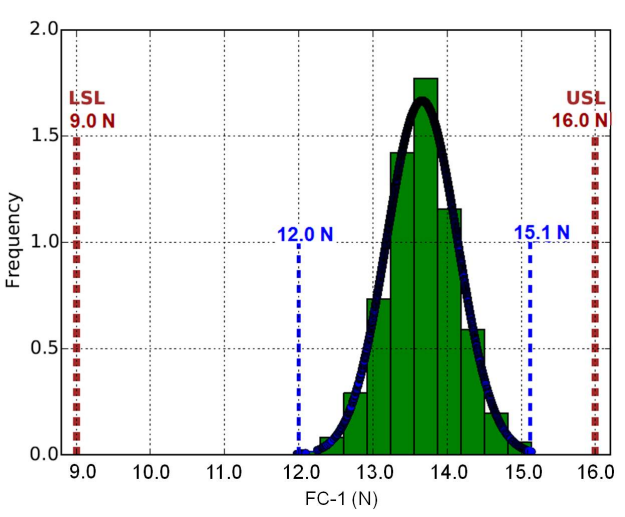

(a) FC-1, $\bar{m}=13.66 \mathrm{~N}, \sigma=0.239$.

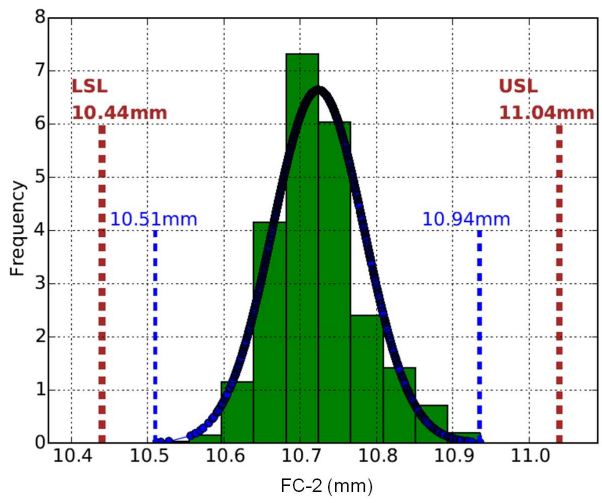

(b) FC-2, $\bar{m}=10.724 \mathrm{~mm}, \sigma=0.060$.

Fig. 16: Dispersion of the functional conditions: FC-1 and FC-2.

function (functional conditions) are computed by simulation. The results are reported in Figures 16, 17 and 18 The

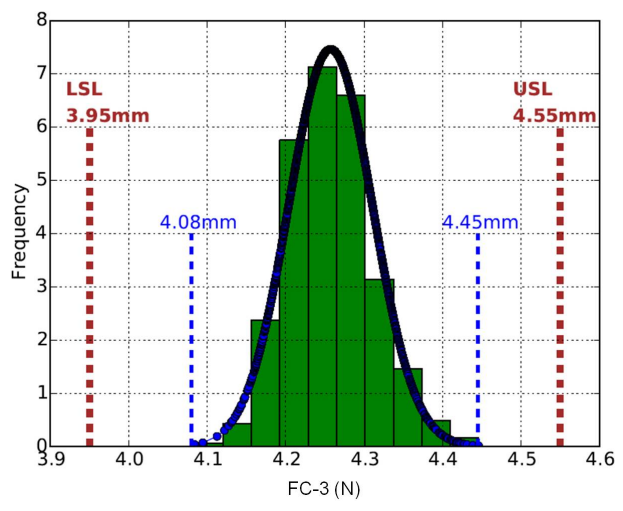

(a) FC-3, $\bar{m}=4.257 \mathrm{~mm}, \sigma=0.053$.

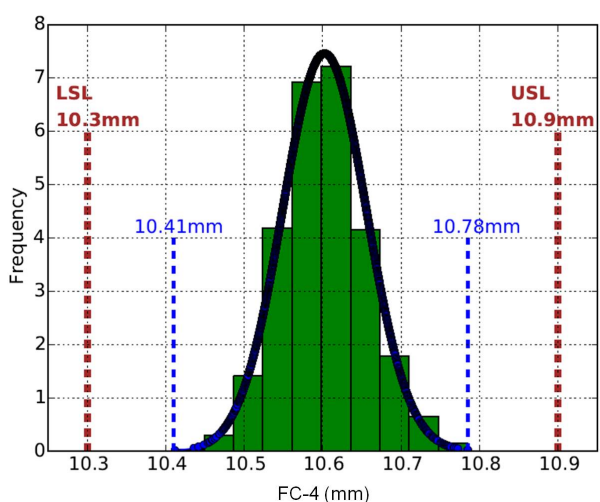

(b) FC-4, $\bar{m}=10.602 \mathrm{~mm}, \sigma=0.054$.

Fig. 17: Dispersion of the functional conditions: FC-3 and FC-4.

\footnotetext{
7 One of the great advantages of the NSCD method, is precisely to allow a drastic decrease of the simulation time (while keeping very good predictability), hence making it possible to run a Monte Carlo method with several thousands of simulations.

${ }^{8}$ https://ciment-grid.ujf-grenoble.fr/
} 
major observations are:

1. In case of the output variable FC-1 (contact force), the statistical distribution is from $[12.0,15.1] \mathrm{N}$ which is well within the limit defined by the USL $(16.0 \mathrm{~N})$ and LSL (9.0N) (see Figure 16(a)). However the distribution is not centered, the index of absolute centering is calculated as $C_{c}=0.3314$, the process capability $C_{p}>1$ and the process capability index is $C_{p k}=1.631$ (see Appendix $\mathrm{A}$ for the definition of these variables). The positive shift in the distribution of the FC-1 ensures good contact pressure between the moving and the fixed contacts, which ultimately helps to reduce the arcing between the contacts. It will help to ensure the safety of the product even in the case of erosion between the contacts.

2. The output variable FC-6 (distance between tripping bar and plunger), is associated with the tripping function of the C-60 breaker. The spread of the statistical distribution is very small and well within the limits, it increases the safety margin of the product. The shift is calculated as $C_{c}=-0.165$, it indicates that the spread is shifted to the left side of the mean value (see Figure $18(\mathrm{~b})$ ). The process capability $C_{p}>1$ and the process capability index is $C_{p k}=3.825$. The $\mathrm{C}-60$ breaker will trip only in the event of abnormal conditions in the circuit. The negative shift in the distribution of the FC-6 and larger value of $C_{p k}$, ensure the minimum gap between the tripping bar and the magnetic plunger. It will help to trip the breaker quickly without any time delay, which is essential for the safety of the electrical network/installations.

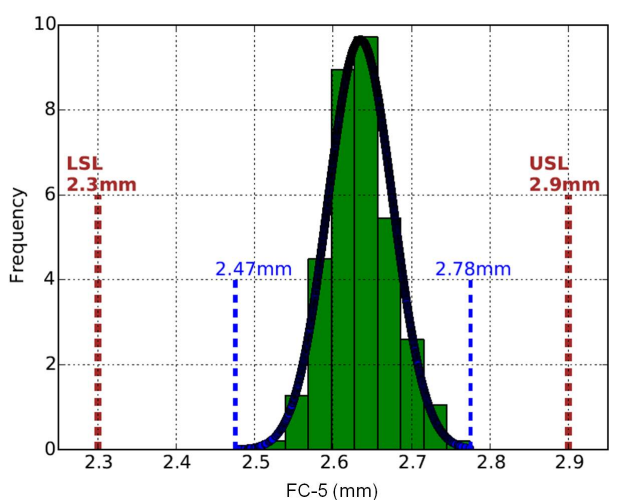

(a) $\mathrm{FC}-5, \bar{m}=2.635 \mathrm{~mm}, \sigma=0.041$

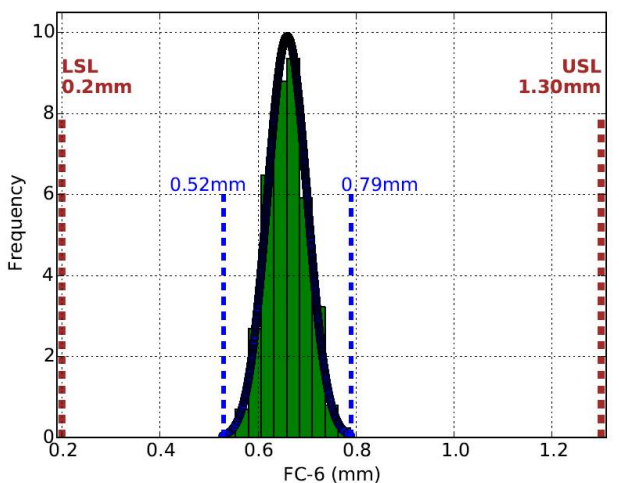

(b) FC-6, $\bar{m}=0.659 \mathrm{~mm}, \sigma=0.040$

Fig. 18: Dispersion of the functional conditions: FC-5 and FC-6.

3. Similarly for the output variables FC-2, FC-3, FC-4 and FC-5 (X, Y coordinates of the pin and lamage center from the needle axis), the statistical distribution is well within the limits (USL and LSL), see Figures 16(b), 17(a)-(b) and 18(a). However the safety margin is lower when compared to the FC-1 and FC-6, see Figures 16,a) and 18,b). The shift in the distribution of FC-2, FC-3, FC-4 and FC-5 is minimal, however the spread $C_{p}$ in the distribution of FC-2 and FC-3 is greater when compared to the FC- 4 and FC-5. It may lead to tripping delays between the pin-side and lamage-side. The process capability index is $C_{p k}>1.5$ which ensures the interconnection/association between the auxiliary and the C-60 breaker.

4. Globally the output variable distribution satisfies the $\pm 4 \sigma$ limit, $C_{p}>1.33$ and $C_{p k}>1.5$, which means that the capability of the output variables is $99.994 \%$ and the rejection rate is $64 \mathrm{PPM}$

\subsection{Conclusions}

The NSCD numerical scheme allows to drastically reduce the simulation time (compared to simulation times obtained with commercial software packages that rely on compliant regularized contact models). This permits in turn to use in a reliable way, a Monte Carlo method which demands a great number of simulations. Monte Carlo analysis would otherwise be very difficult (if not impossible) to obtain. A sensitivity analysis of the C-60 mechanism is thus doable. From the Monte-Carlo simulation, it can be concluded that for the batch-wise and

\footnotetext{
9 Parts-Per-Million, is the fraction of quantity per one million parts.
} 
global production of the C-60 breaker, a joint clearance of $[0.01,0.13] \mathrm{mm}$ (see Figure 15 ) will ensure the robust performance.

\section{Experimental validation}

In this section we report comparisons between numerical results obtained with the NSCD method (sections 2 and 2.4, and experimental data obtained on a physical prototype built by Schneider Electric. Referring to the two arrows in Figure 11, the comparisons are made by recording force/displacement histories at the moving contact, and at the tripping bar. The rationale behind this choice, is that measuring physical quantities inside such a mechanism is a rather uneasy task in general. These two force/displacement histories are of interest for Schneider Electric, and they represent non-trivial characteristic features of the circuit breaker mechanism. They are therefore quite suitable for the validation of the numerical results.

\subsection{Experimental set-up}

Physical prototypes of the C-60 breaker have been built for the validation of the C-60 numerical simulation model. Clearance in the revolute joints can be modified either by modifying the bearing diameter, or by modifying the journal diameter. One of the simplest methods for modifying the bearing or journal diameter is by the machining process. However in the C-60 mechanism most of the parts are plastic molded and the machining process cannot be used. Also it is much easier to change the diameter of the bearing when compared to the journal diameter. The alternative method is to modify the molds (core and cavity). To change the diameter of the bearing, the core pins must be changed to the suitable diameter. This method is very time consuming and expensive. However it will allow to change the dimensions of the hole/cavity to the required accuracy. In our case we need the tolerance to be as small as possible (NFT58000, precision class [19]). All other parts of the C-60 mechanism are reused (without modification) from the manufacturing batch. The functional dimensions of all the mechanism parts are measured and recorded. The first step is to record all the associated functional dimensions of the parts. In the second step, the test bench is prepared and calibrated for the test. The test bench consists of the fixture to mount the C-60 breaker

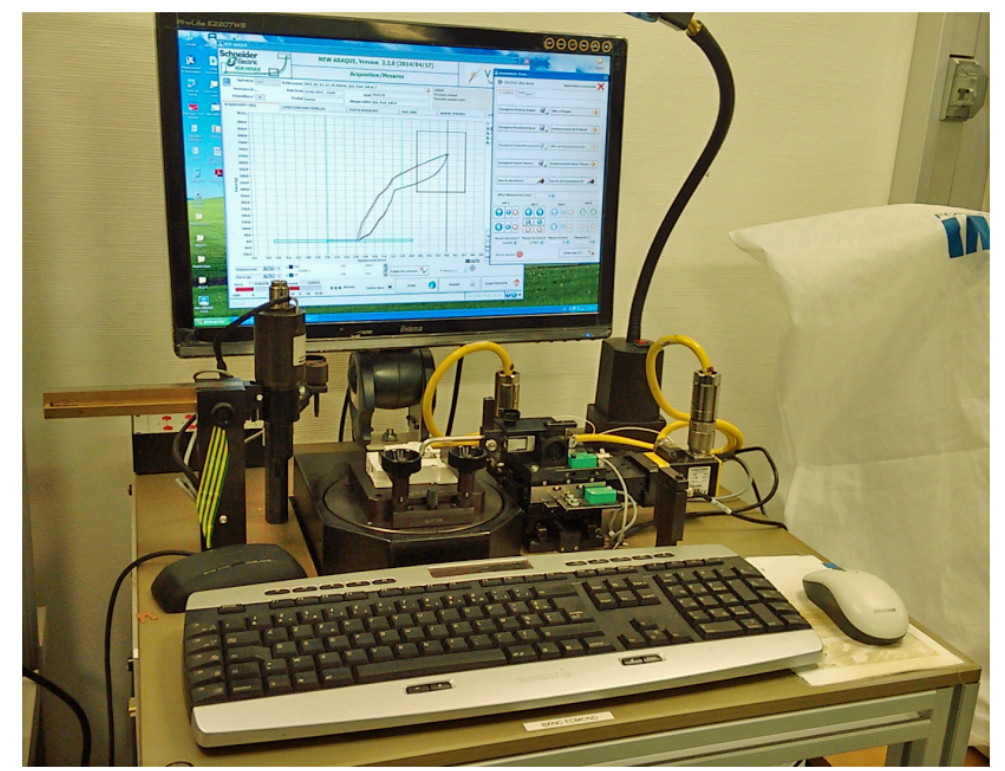

Fig. 19: Experimental test bench for contact/tripping force measurement.

and the moving table which comprises a pair of linear motion guide, see Figure 19 . Each guide unit is composed of a guide rail and a slider is mounted on the guide rail. The bi-axial movement is given to the moving table through the slider. The probe is attached to the load cell and mounted on the moving table. The bi-axial movement of the 
moving table is measured by two position sensors. The uniform bi-axial motion of the test bench is regulated by a controller and all the system is connected to the CPU and controlled through a specific computer program.

Table 7: Prototype : Radial clearance in the revolute joints.

\begin{tabular}{|c|c|}
\hline Name of the joint & $\begin{array}{c}\text { Radial clearance in the joint } \\
\text { Measured value }(\mathrm{mm})\end{array}$ \\
\hline$J_{1}$ & 0.085 \\
\hline$J_{2}$ & 0.05 \\
\hline$J_{3}$ & 0.06 \\
\hline$J_{4}$ & 0.06 \\
\hline$J_{5}$ & 0.045 \\
\hline$J_{6}$ & 0.045 \\
\hline$J_{7}$ & 0.055 \\
\hline
\end{tabular}

\subsection{Contact force versus displacement}

A regulated linear velocity $(0.5 \mathrm{~mm} / \mathrm{s})$ is imposed to the probe of the load cell through the moving table. The contact force of the moving contact $C_{7}$ (see Figure 1 ) is measured with the help of load cell, and is recorded by the computer program. At the reference position the C-60 mechanism is allowed to reach the static equilibrium and the value of the peak force recorded. After the static equilibrium position has been reached, the plunger/probe is moved from the reference position to the initial position of the probe by changing the direction of the velocity to $-0.5 \mathrm{~mm} / \mathrm{s}$ (backward motion). The backward motion is continued till the initial position (where the load on the plunger/probe becomes zero). We have followed a similar methodology for the virtual testing (virtual test bench) of the C-60 product using the simulation.

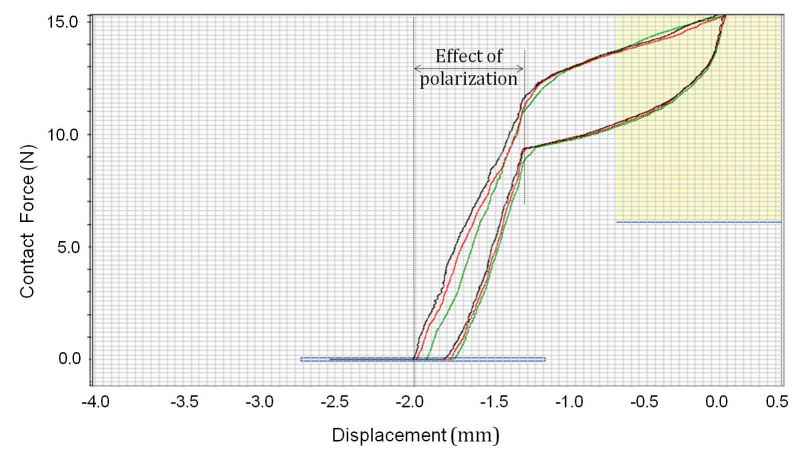

(a) Experimental result.

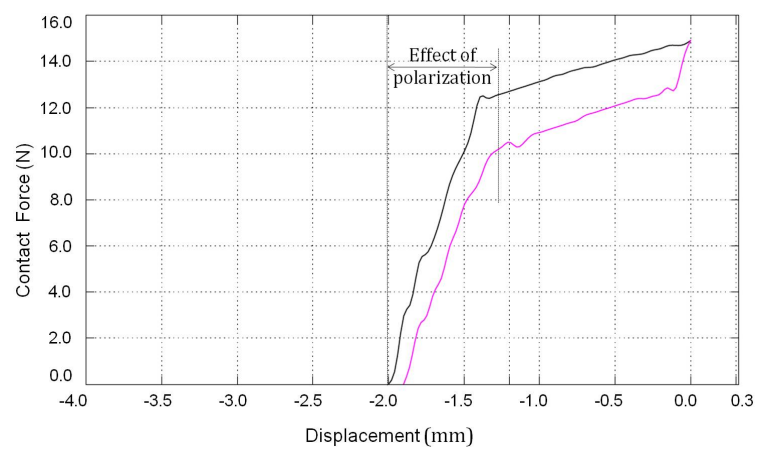

(b) Simulation result.

Fig. 20: Contact force versus displacement.

The major conclusions are:

1. The effect of polarization ${ }^{10}$ of the joints is approximately less by $50 \%$ when compared to the total displacement of the moving contact. At the static equilibrium (at the end of forward motion) of the sample, the recorded contact force is $15.28 \mathrm{~N}$ and the total displacement is $2.0 \mathrm{~mm}$, see Figure 20 (a). The results of the experimental test are compared with the numerical simulation. In case of the numerical test the effect of polarization is similar to that of the experimental test, see Figure 20(b). However the trajectory of the contact force in both the experimental and numerical tests are not identical, this may be due to the fact that the geometrical variations on the contacting surfaces of the parts are not considered in the case of numerical simulation. In reality the geometrical variations always exist and these variations may change the polarization of the parts (contact points between the parts). In case of the numerical simulation, the contact force at the static equilibrium is $14.96 \mathrm{~N}$. The percentage relative error in the contact force between the experimental and numerical tests is $2.08 \%$.

2. The trajectory of the contact force in case of forward motion is lagging behind the backward motion of the moving contact. This is due to the effects of friction (change in the direction of the frictional forces) in the

\footnotetext{
10 See Definition 1
} 
joints. In case of the numerical simulation, the coefficient of friction between the plastic-plastic materials is considered to be $\mu=0.3$, and between the steel-plastic materials $\mu=0.1^{11}$. In case of experimental test the real values of the coefficient of friction are not known. This may be one of the reasons behind the slightly different behaviour of the contact force trajectories between the experiment and virtual test.

\subsection{Tripping force versus displacement}

The circular cavity on the tripping bar is used for the application of external force to trip the product (see Figure 1 ). The tripping operation is possible only if the product is in ON condition. In the case of pin-side tripping, the

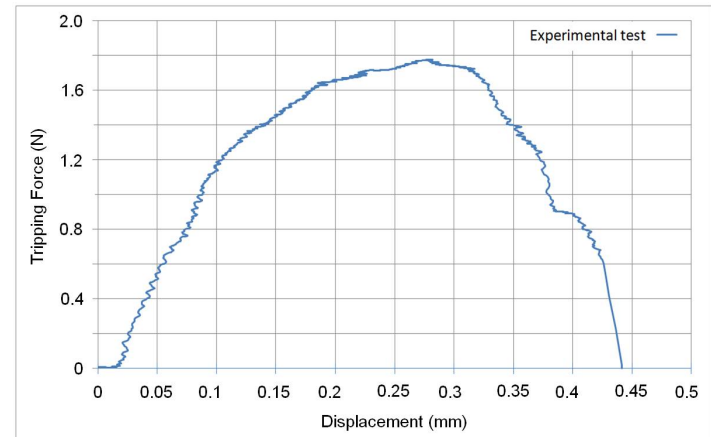

(a) Experimental test

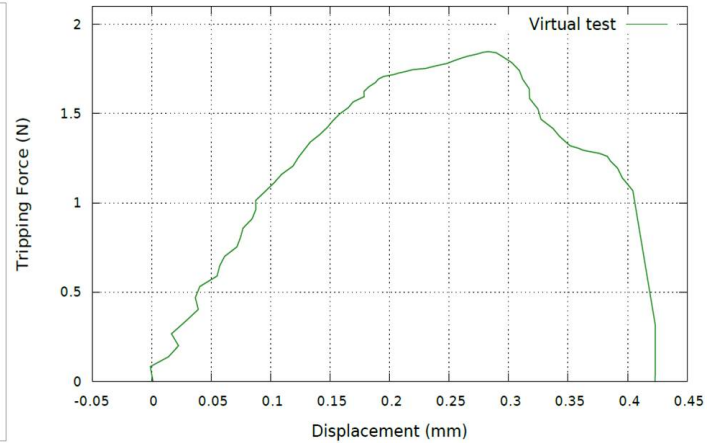

(b) Virtual (numerical) test

Fig. 21: Tripping force vs displacement: pin-side.

position of the cavity on the tripping bar is recorded by the plunger/probe and set as the reference position. When the plunger/probe comes in contact with the tripping bar cavity, a small load is detected by the load cell. This is the starting point of the test. The programmed controller regulates a linear motion velocity of $0.5 \mathrm{~mm} / \mathrm{s}$ for the plunger through the moving table and the constant velocity is ensured by the controller. The tripping bar is pushed forward (in positive $\mathrm{Y}$ direction) to break the frictional contact between the hook and the tripping bar $C_{4}$ and the corresponding distance, and the tripping force is recorded by the position sensor and the load cell respectively, see Figure 19. The numerical tests are carried in a similar way. For the numerical simulation, we have performed some iterations to find out the approximate location of the potential contact points similar to the prototype model. In doing so, the experimental results helped us to predict the possible contacts in the hook and tripping bar joint. Also we have captured the magnified images of the contact surfaces to understand the profile of the surface. All these inputs are used to locate approximately the potential contacts in the hook and tripping bar contact. The major conclusions are:

1. In case of the experimental results, the recorded peak force is $1.77 \mathrm{~N}$ at a distance of $0.27 \mathrm{~mm}$. The total trip distance is $0.44 \mathrm{~mm}$, see Figure 21 (a). In case of the numerical simulation, the trajectory of the tripping force is slightly different, see Figure 21(b). The total trip distance is $0.42 \mathrm{~mm}$, with a variation of $0.02 \mathrm{~mm}$ between the numerical and experimental results. However the peak force in case of the numerical test is $1.87 \mathrm{~N}$ and when compared to the experimental results the variation is $0.1 \mathrm{~N}$, see Figures 21.a)-(b).

2. The interaction between the tripping bar and hook is defined by plane-plane contact. The contacting surface profile will define the potential contact points on the entire surface. During the mass production, the trajectory of the tripping force may vary slightly based on the location of the contact points. This is evident from the simulation and experimental results as depicted in the Figure 21(a)-(b). After a careful study of the tripping force trajectory on the mass production samples, it is observed that the number of contacts may vary from sample to sample, and the tripping force varies slightly.

We can conclude that the NSCD scheme and the modelling choices made in section 3, allow us to predict in a very satisfactory way these two important force/displacement histories in the C-60 mechanism.

\footnotetext{
11 The variety of materials and the poor knowledge of their properties, totally preclude the use of friction models that involve dynamics and many parameters [41] whose physical meaning is unclear [74], in this type of industrial application.
} 


\section{Conclusions}

This article is devoted to the numerical simulation of the C-60 circuit breaker built by Schneider Electric, taking into account the influence of the clearances in joints on the functional conditions. Spatial (3D) revolute joints are modelled with both radial and axial clearances taking into account contact with flanges. Unilateral contact, Coulomb's friction and Newton impact laws are modeled within the framework of nonsmooth mechanics without resorting to some regularizations or compliance/damping at contact. The nonsmooth contact dynamics method based on an event-capturing time-stepping scheme with a second order cone complementarity solver is used to perform the numerical integration. The salient conclusions of the paper are as follows:

- The nonsmooth modeling approach [4] together with an event-capturing time-stepping scheme allows us to simulate, in an efficient and robust way, the contact and impact phenomena that occur in joints with clearances. Furthermore, the stabilization of the constraints at the position level, made through the stabilized combined projected Moreau-Jean scheme [2] enables to study mechanisms with tight clearances.

- It is shown that the method allows to perform a sensitivity analysis, based on Monte-Carlo technique, of the mechanism with respect to joint clearances, due to simulation times which are drastically reduced compared with other contact models and numerical schemes (smoothed or event-capturing schemes).

- The use of boundary representation (B-rep) and CAD libraries allows us to take into account complex contact geometries without using closed form formulas for the gap distance and the local frame at contact. This enables to deal with real geometries in industrial applications directly obtained from the mechanical design.

- The sensitivity of results with respect to contact parameters is rather weak. This aspect is quite important when dealing with parameters which are difficult to measure and sensitive to environmental conditions such as the coefficient of friction. It appears that in the modeling of circuit breakers mechanisms, a good modeling of the threshold effect depending on the normal contact force with perfect stiction that is inherent to the Coulomb friction model is the most important phenomena, rather than the accurate magnitude of the friction coefficient. The same conclusion can be drawn for the coefficient of restitution when the impacts are almost plastic. Finally, with this approach, we avoid the dispersion of results due to compliance contact model often reported in the literature [26, 24, 10].

- Detailed comparisons with experimental data obtained at the Schneider Electric laboratory, prove the very good prediction capabilities of the nonsmooth approach for the C60 breakers in an industrial context. Especially, this study allows design engineers to understand what are the critical parts in the mechanism that need to be manufactured with an high precision class. On the contrary, some parts are not critical for the functional conditions of the mechanisms and may lead to some reduction of the manufacturing cost.

\section{A Process capability and performance indices}

If worst-case tolerances are specified for the inputs, a worst-case tolerance can be calculated for the output. Let us denote the output by $Y$ and the inputs by $X_{i}, i \in(1, \ldots, n)$. We consider, $Y=f\left(X_{i}\right)$ and that the inputs have worst-case tolerances $N_{X i} \pm T_{X i}$. Then the resulting worst-case tolerance for $Y$ is denoted as: $N_{Y} \pm T_{Y}$. The lower specification limit (LSL), the upper specification limit (USL), nominal (N) and the tolerance is calculated as:

$$
\begin{aligned}
& \mathrm{LSL}_{Y}=\min _{N_{X i}-T_{X i} \leqslant X_{i} \leqslant N_{X i}+T_{X i}} f\left(X_{i}\right) \\
& \\
& \mathrm{USL}_{Y}=1, . ., n \max _{N_{X i}-T_{X i} \leqslant X_{i} \leqslant N_{X i}+T_{X i}} f\left(X_{i}\right) \\
& i=1, . ., n \\
& N_{Y}=\left(\frac{\mathrm{LSL}_{Y}+\mathrm{USL}_{Y}}{2}\right) \\
& T_{Y}=\left(\frac{\mathrm{USL}_{Y}-\mathrm{LSL}_{Y}}{2}\right)
\end{aligned}
$$

The most common way of denoting the statistical tolerance is $N_{X} \pm T_{X}<S T>$. The statistical tolerance is always associated with a mean value $(\bar{m})$, and the standard deviation $(\sigma)$. The tolerance range is given as: $T_{X}=3 \sigma$. The statistical tolerances are more restrictive. They further requires the process capability $\left(C_{p}\right)$ and process capability index $\left(C_{p k}\right)$ to be centered for a good process [38 3960]. 


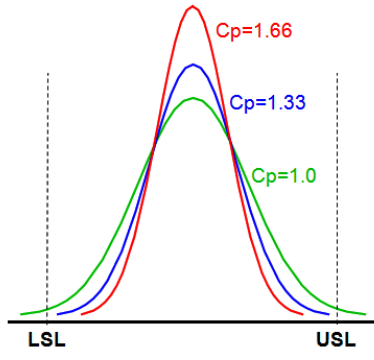

(a) Variation in $C_{p}$.

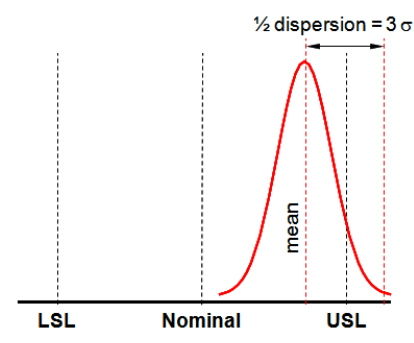

(b) Measure of $C_{p k}$.

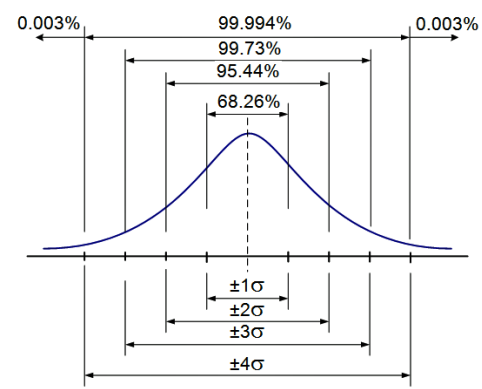

(c) Capability/rejection for different $\sigma$.

Fig. 22: Process capability $C_{p}$ and process capability index $C_{p k}$.

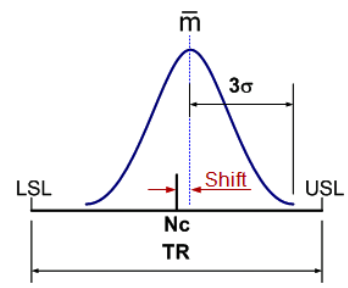

(a) Right centered process.

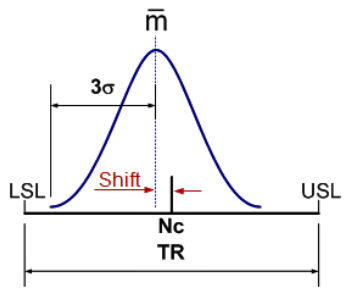

(b) Left centered process.

Fig. 23: Mean shift between desired (design) and actual (process) $C_{c}$.

Process capability $\left(C_{p}\right)$ : It evaluates the tolerance Range (TR) compared to a standard six-sigma $(6 \sigma)$ production dispersion (see Figure 22 a)). The $C_{p}$ measures how close a process is running to its specification limits, relative to the natural variability of the process (normally it is given by $6 \sigma$ )

$$
C_{p}=\frac{\text { Tolerance Range }(\mathrm{TR})}{6 \sigma}=\frac{\mathrm{USL}-\mathrm{LSL}}{6 \sigma}
$$

Process capability index $\left(C_{p k}\right)$ : It evaluates, if the specification can be met given the process spread and tool shift from nominal. In other word, $C_{p k}$ is the capability of the process to see whether or not the mean is centered between the specification limits (see Figures 22 b) and 23 a)-(b)).

$$
C_{p k}=\min \left(\frac{\mathrm{USL}-\bar{m}}{3 \sigma}, \frac{\bar{m}-\mathrm{LSL}}{3 \sigma}\right)
$$

where $\bar{m}$ is the mean value. An index of absolute centering $C_{c}$ quantifies the shift of mean from the Nominal Value, see Figure 23 a)-(b). It is written as a ratio:

$$
C_{c}=\frac{\text { Shift }}{0.5 \mathrm{TR}}
$$

\section{References}

1. M. Abadie. Dynamic simulation of rigid bodies: Modelling of frictional contact. In B. Brogliato, editor, Impacts in Mechanical Systems: Analysis and Modelling, volume 551 of Lecture Notes in Physics (LNP), pages 61-144. Springer, 2000.

2. V. Acary. Projected event-capturing time-stepping schemes for nonsmooth mechanical systems with unilateral contact and Coulomb's friction. Computer Methods in Applied Mechanics and Engineering, 256:224-250, 2013.

3. V. Acary, O. Bonnefon, M. Brémond, O. Huber, F. Pérignon, and S. Sinclair. Siconos: a software for the modeling, the simulation and the control of nonsmooth dynamical systems. http://siconos.gforge.inria.fr. 2005-2016.

4. V. Acary and B. Brogliato. Numerical Methods for Nonsmooth Dynamical Systems. Applications in Mechanics and Electronics, volume 35 of Lecture Notes in Applied and Computational Mechanics. Springer Verlag, Berlin Heidelberg, 2008.

5. V. Acary and F. Cadoux. Recent Advances in Contact Mechanics, Stavroulakis, G. E. (Ed.), volume 56 of Lecture Notes in Applied and Computational Mechanics, chapter Applications of an existence result for the Coulomb friction problem. Springer Verlag, 2013.

6. V. Acary, F. Cadoux, C. Lemaréchal, and J. Malick. A formulation of the linear discrete Coulomb friction problem via convex optimization. ZAMM-Journal of Applied Mathematics and Mechanics/Zeitschrift für Angewandte Mathematik und Mechanik, 91(2):155-175, 2011.

7. V. Acary and F. Pérignon. An introduction to Siconos. Technical Report RT-0340, INRIA, 2007.

8. N. Akhadkar, V. Acary, and B. Brogliato. Analysis of collocated feedback controllers for four-bar planar mechanisms with joint clearances. Multibody System Dynamics, 38(2):101-136, 2016. 
9. O.A. Bauchau and J. Rodriguez. Modeling of joints with clearance in flexible multibody systems. International Journal of Solids and Structures, 39(1):41-63, 2002.

10. M. A. Ben Abdallah, I. Khemili, and N. Aifaoui. Numerical investigation of a flexible slider-crank mechanism with multijoints with clearance. Multibody System Dynamics, 38(2):173-199, 2016.

11. B. Brogliato. Nonsmooth Mechanics: Models, Dynamics and Control. Springer International Publishing, Switzerland, 3rd edition, 2016.

12. O. Brüls, V. Acary, and A. Cardona. Simultaneous enforcement of constraints at position and velocity levels in the nonsmooth generalized$\alpha$ scheme. Computer Methods in Applied Mechanics and Engineering, 281:131-161, 2014.

13. C. Brutti, G. Coglitore, and P.P. Valentini. Modeling 3D revolute joint with clearance and contact stiffness. Nonlinear Dynamics, 66(4):531-548, 2011.

14. K.W. Chase and W.H. Greenwood. Design issues in mechanical tolerance analysis. Manufacturing Review, 1(1):50-59, 1988.

15. K.W. Chase and A.R. Parkinson. A survey of research in the application of tolerance analysis to the design of mechanical assemblies. Research in Engineering design, 3(1):23-37, 1991.

16. Y. Chen, Y. Su, and C. Chen. Dynamic analysis of a planar slider-crank mechanism with clearance for a high speed and heavy load press system. Mechanism and Machine Theory, 98:81 - 100, 2016.

17. OpenCascade Corp. http: / /www. opencascade.org. 2016.

18. J.Y. Dantan and A.J. Qureshi. Worst-case and statistical tolerance analysis based on quantified constraint satisfaction problems and Monte Carlo simulation. Computer-Aided Design, 41(1):1-12, 2009.

19. Association Francaise de Normalisation. NFT58000 Plastics - tolerances applicable to moulded plastic parts, 1987.

20. G. De Saxcé. Une généralisation de l'inégalité de Fenchel et ses applications aux lois constitutives. Comptes Rendus de l'Académie des Sciences, t 314, série II:125-129, 1992.

21. J. F. Deck and S. Dubowsky. On the limitations of predictions of the dynamic response of machines with clearance connections. J. Mech. Des., 116(3):833-841, 1994.

22. S.G. Dhande and J. Chakraborty. Mechanical error analysis of spatial linkages. Journal of Mechanical Design, 100(4):732-738, 1978.

23. S. Dubowsky, J.F. Deck, and H. Costello. The dynamic modeling of flexible spatial machine systems with clearance connections. J. Mech., Trans., and Automation, 109(1):87-94, 1987.

24. P. Flores. A parametric study on the dynamic response of planar multibody systems with multiple clearance joints. Nonlinear Dynamics, 61(4):633-653, 2010.

25. P. Flores and J. Ambrósio. Revolute joints with clearance in multibody systems. Computers \& Structures, 82(17):1359-1369, 2004.

26. P. Flores, J. Ambrósio, and J.P. Claro. Dynamic analysis for planar multibody mechanical systems with lubricated joints. Multibody System Dynamics, 12(1):47-74, 2004.

27. P. Flores, R. Leine, and C. Glocker. Modeling and analysis of planar rigid multibody systems with translational clearance joints based on the non-smooth dynamics approach. Multibody System Dynamics, 23:165-190, 2010.

28. R.E. Garrett and A.S. Hall. Effect of tolerance and clearance in linkage design. Journal of Manufacturing Science and Engineering, 91(1):198-202, 1969.

29. W.H. Greenwood and K.W. Chase. Worst case tolerance analysis with nonlinear problems. Journal of Manufacturing Science and Engineering, 110(3):232-235, 1988.

30. A. Gummer and B. Sauer. Influence of contact geometry on local friction energy and stiffness of revolute joints. Journal of Tribology, 134(2):021402, 2012.

31. A. Gummer and B. Sauer. Modeling planar slider-crank mechanisms with clearance joints in RecurDyn. Multibody System Dynamics, 31:127-145, 2014.

32. A.F. Haroun and S.M. Megahed. Simulation and experimentation of multibody mechanical systems with clearance revolute joints. In Proceedings of World Academy of Science, Engineering and Technology. World Academy of Science, Engineering and Technology, 2012.

33. X. Huang and Y. Zhang. Robust tolerance design for function generation mechanisms with joint clearances. Mechanism and Machine Theory, 45(9):1286-1297, 2010.

34. M. Jean. The non-smooth contact dynamics method. Computer Methods in Applied Mechanics and Engineering, 177(3):235-257, 1999.

35. M. Jean and J.J. Moreau. Dynamics in the presence of unilateral contacts and dry friction: a numerical approach. In G. del Piero and F. Maceri, editors, Unilateral Problems in Structural Analysis II, number 304 in CISM Courses and Lectures, pages 151-196. Springer Verlag, 1987.

36. A. Jeang. Tolerance design: choosing optimal tolerance specifications in the design of machined parts. Quality and Reliability Engineering International, 10(1):27-35, 1994.

37. T. Kakizaki, J.F. Deck, and S. Dubowsky. Modeling the spatial dynamics of robotic manipulators with flexible links and joint clearances. J. Mech. Des., 115(4):839-847, 1993.

38. V.E. Kane. Process capability indices. Journal of Quality Technology, 18(1):41-52, 1986.

39. S. Kotz and N.L. Johnson. Process Capability Indices. CRC Press, 1993.

40. A. Krinner and T. Thümmel. Non-smooth behaviour of a linkage mechanism with revolute clearance joints. In New Advances in Mechanisms, Transmissions and Applications, pages 233-241. Springer, 2014.

41. V. Lampaert, J. Swevers, and F. Al-Bender. Modification of the Leuven friction model structure. IEEE Transactions on Automatic Control, 47(4):683-687, 2002.

42. H.M. Lankarani and P.E. Nikravesh. Continuous contact force models for impact analysis in multibody systems. Nonlinear Dynamics, 5(2):193-207, 1994.

43. C. Lemaréchal. Using a MODULOPT minimization code. Unpublished Technical Note, INRIA Rocquencourt, 1980. http://people.sc.fsu.edu/ inavon/5420a/modulopt.pdf.

44. C. Lemaréchal and E. Panier. Les modules M2QN1 et MQHESS. Unpublished Technical Note, INRIA Rocquencourt, 1983. https://who.rocq.inria.fr/Jean-Charles.Gilbert/modulopt/optimization-routines/m2qn1/m2qn1.pdf. 
45. C. Liu, Q. Tian, and H. Hu. Dynamics and control of a spatial rigid-flexible multibody system with multiple cylindrical clearance joints. Mechanism and Machine Theory, 52:106-129, 2012.

46. C. Liu, K. Zhang, and L. Yang. The compliance contact model of cylindrical joints with clearances. Acta Mechanica Sinica, 21(5):451-458, 2005 .

47. F. Marques, F. Isaac, N. Dourado, and P. Flores. An enhanced formulation to model spatial revolute joints with radial and axial clearances. Mechanism and Machine Theory, 116:123 - 144, 2017.

48. J.J. Moreau. Unilateral contact and dry friction in finite freedom dynamics. In J.J. Moreau and P.D. Panagiotopoulos, editors, Nonsmooth Mechanics and Applications, number 302 in CISM, Courses and lectures, pages 1-82. CISM 302, Spinger Verlag, Wien- New York, 1988.

49. J.J. Moreau. Some numerical methods in multibody dynamics: Application to granular materials. Eur. J. Mech /A. Solids, supp.(13):93$114,1994$.

50. J.J. Moreau. Numerical aspects of the sweeping process. Computer Methods in Applied Mechanics and Engineering, 177:329-349, 1999.

51. S.D. Nigam and J.U. Turner. Review of statistical approaches to tolerance analysis. Computer-Aided Design, 27(1):6-15, 1995.

52. J.C. García Orden. Analysis of joint clearances in multibody systems. Multibody system dynamics, 13(4):401-420, 2005.

53. C. Pereira, A. Ramalho, and J. Ambrosio. An enhanced cylindrical contact force model. Multibody System Dynamics, 35(3):277-298, 2015.

54. C.M. Pereira, A.R. Ramalho, and J. Ambrosio. A critical overview of internal and external cylinder contact force models. Nonlinear Dynamics, 63:681-697, 2011.

55. C.M. Pereira, A.R. Ramalho, and J. Ambrosio. An enhanced cylindrical contact force model. Multibody System Dynamics, 35(3):277-298, 2015.

56. PythonOCC. 3D CAD/CAE/PLM development framework for the Python programming language. http://www.pythonocc.org 2016.

57. E. Salahshoor, S. Ebrahimi, and M. Maasoomi. Nonlinear vibration analysis of mechanical systems with multiple joint clearances using the method of multiple scales. Mechanism and Machine Theory, 105:495 - 509, 2016.

58. F. Scholz. Tolerance stack analysis methods. Research and Technology Boeing Information \& Support Services, Boeing, Seattle, pages $1-44,1995$.

59. V.J. Skowronski and J.U. Turner. Using Monte-Carlo variance reduction in statistical tolerance synthesis. Computer-Aided Design, 29(1):63-69, 1997.

60. V. Srinivasan. Iso deliberates statistical tolerancing. In Geometric Design Tolerancing: Theories, Standards and Applications, pages 77-87. Springer, 1998.

61. C. Studer. Numerics of Unilateral Contacts and Friction. - Modeling and Numerical Time Integration in Non-Smooth Dynamics, volume 47 of Lecture Notes in Applied and Computational Mechanics. Springer Verlag, 2009.

62. S.Yan, W. Xiang, and L. Zhang. A comprehensive model for 3D revolute joints with clearances in mechanical systems. Nonlinear Dynamics, 80(1):309-328, 2015.

63. T. Thümmel and K. Funk. Multibody modelling of linkage mechanisms including friction, clearance and impact. In Proceedings of the 10th World Congress on the Theory of Machines and Mechanisms in Oulu, June 20 to 24, volume 4, pages 1387-1392. Oulu University Press, Finland, 1999.

64. N.T. Thomopoulos. Essentials of Monte Carlo simulation: Statistical methods for building simulation models. Springer Science \& Business Media, 2012.

65. T. Thümmel. Experimentelle Mechanismendynamik: Messung, Modellierung, Simulation, Verifikation, Interpretation und Beeinflussung typischer Schwingungsphänomene an einem Mechanismenprüfstand. PhD thesis, München, Technische Universität München, Habil.-Schr., 2012.

66. T. Thümmel and L. Ginzinger. Measurements and simulations of a crank and rocker mechanism including friction, clearance and impacts. In In Proceedings of the IX. International Conference on the Theory of Machines and Mechanisms in Liberec/Czech Republic, Aug.31Sept.2004, pages 763-768. Technical University of Liberec, Department of Textile Machine Design, 2004.

67. T. Thümmel and M. Roßner. Introduction to modelling and parameter identification methodology of linkages by measurements and simulation. In Proceedings of the 13th World Congress in Mechanism and Machine Science, Guanajuato, Mexico, 19-25 June, volume IMD-123, 2011.

68. T. Thümmel, J. Rutzmoser, and H.Ulbrich M. Robner. Friction modeling and parameter value estimation of mechanisms. In The 2nd Joint International Conference on Multibody Systems Dynamics, May 29-June 1,2012, Stuttgart, Germany, pages 302-312. University of Stuttgart, Institute of Engineering and Computational Mechanics, 2012.

69. Q. Tian, C. Liu, M. Machado, and P. Flores. A new model for dry and lubricated cylindrical joints with $\hat{A}$ ăclearance in spatial flexible multibody systems. Nonlinear Dynamics, 64(1):25-47, 2011.

70. Q. Tian, J. Lou, and A. Mikkola. A new elastohydrodynamic lubricated spherical joint model for rigid-flexible multibody dynamics. Mechanism and Machine Theory, 107:210 - 228, 2017.

71. Q. Tian, Q. Xiao, Y. Sun, H. Hu, H. Liu, and P. Flores. Coupling dynamics of a geared multibody system supported by elastohydrodynamic lubricated cylindrical joints. Multibody System Dynamics, 33(3):259-284, 2015.

72. J.U. Turner and M.J. Wozny. Tolerances in computer-aided geometric design. The Visual Computer, 3(4):214-226, 1987.

73. Geoffrey Virlez, Olivier Brüls, Emmanuel Tromme, and Pierre Duysinx. Modeling joints with clearance and friction in multibody dynamic simulation of automotive differentials. Theoretical and Applied Mechanics Letters, 3(1):013003, 2013.

74. M. Wojtyra. Modeling of static friction in closed-loop kinematic chains-uniqueness and parametric sensitivity problems. Multibody System Dynamics, 39(4):337-361, 2017.

75. F. Wu, J.Y. Dantan, A. Etienne, A. Siadat, and P. Martin. Improved algorithm for tolerance allocation based on Monte Carlo simulation and discrete optimization. Computers \& Industrial Engineering, 56(4):1402-1413, 2009.

76. Sadeq Yaqubi, Morteza Dardel, Hamidreza Mohammadi Daniali, and Mohammad Hassan Ghasemi. Modeling and control of crank-slider mechanism with multiple clearance joints. Multibody System Dynamics, 36(2):143-167, 2016. 
77. C. Zhang, J. Luo, and B. Wang. Statistical tolerance synthesis using distribution function zones. International Journal of Production Research, 37(17):3995-4006, 1999.

78. C.C. Zhang and H-P.B. Wang. Robust design of assembly and machining tolerance allocations. IIE transactions, 30(1):17-29, 1997.

79. Z. Zhang, L. Xu, P. Flores, and H.M. Lankarani. A Kriging Model for dynamics of mechanical systems with revolute joint clearances. Journal of Computational and Nonlinear Dynamics, 9(3):031013, 2014. 